\title{
Response of the streaks, the active and passive eddies in an unsteady channel flow
}

\author{
F. Sedat Tardu *, Philippe Vezin \\ Laboratoire des Ecoulements $G^{\prime}$ eophysiques et Industriels, B.P. 53 X, Grenoble C'edex 38041, France
}

\begin{abstract}
Spanwise space-time correlations of the wall shear stress and the longitudinal velocity fluctuations in the low buffer layer of an unsteady channel flow are reported. The imposed amplitude is $20 \%$ of the centerline velocity and the imposed frequency covers a large range going from the quasi-steady limit to the bursting frequency of the corresponding steady flow. The unsteady spanwise correlation coefficient is investigated both through its own modulation characteristics (amplitude and phase shifts) and those of the resulting streak spacing. A good correspondence is found between the modulation of the streak spacing and that of the ejection period. The data is further analyzed by temporal filtering of the wall shear stress and streamwise velocity fluctuations. It is shown that the large outer-layer structures play a "passive" role in the unsteady response of the near wall turbulence. The inner wall eddies, in return, are amply responsible for the unsteady reaction of both the turbulent wall shear stress and the streamwise velocity intensities in the buffer layer.
\end{abstract}

\section{Introduction}

Turbulent shear flows with periodically forced freestream velocity have been intensively investigated during the last two decades. The pipe flow experiments of $\mathrm{Tu}$ and Ramaprian (1983) and of the Illinois team (Mao and Hanratty, 1986; Finnicum and Hanratty, 1988), the unsteady boundary layer investigation of Brereton et al. (1990) and the channel flow experiments conducted by the Grenoble group (Tardu and Binder, 1993; Tardu et al., 1994) could be representative of yet an incomplete list of recent research on this topic. The practical importance of forced unsteady turbulent layers only partly explains the increasing interest in this subject. The fundamental aspects are more attractive. The forcing provides an effective way of determining the "frequency response" of the near wall turbulence. The oscillating shear acts on the near wall flow viewed as a system. The

\footnotetext{
* Corresponding author. Tel.: +33-4-76827030; fax: +33-476825271

E-mail address: sedat.tardu@hmg.inpg.fr (F. Sedat Tardu).
}

sensing of the state (outputs) through the modulation characteristics of the flow quantities, determines its transfer function.

The temporal nonequilibrium sometimes dictates an unexpected response of the near wall turbulence. First of all, the imposed frequency range demarcating the quasisteady regime differs from one quantity to another. There is a large consensus on the existence of a relaxation regime, which is subsequently set-up. Roughly speaking, when the imposed unsteadiness is rapid, the modulation of the turbulent quantities weakens and large time lags with respect to the centerline velocity appear. In the models which are more or less inspired from the rapid distortion theory, these behaviors may be qualitatively predicted by taking into account an effective strain (Mankbadi and Liu, 1992; Tardu and Da Costa, 2001), an effective pressure gradient (Mao and Hanratty, 1986) or an effective oscillating shear (Tardu and Binder, 1997), through a first order response relationships with constant time scales. These models fail in a third regime characterized by imposed frequencies significantly larger than the bursting frequency and wherein the modulation of turbulent quantities increases (Finnicum and Hanratty, 1988; Feng et al., 1993). 
Most of the past research on the response of the near wall turbulence has been devoted to the modulation characteristics of the Reynolds shear stresses and to the wall shear stress intensity. Only limited attention has been paid for the length scales. The time-space transverse correlations of the fluctuating wall shear stress reported by Finnicum and Hanratty (1988) are the only available probe data to our knowledge. These authors have determined the cyclic variations of the spanwise characteristic scale of wall turbulence, i.e., the streak spacing $\langle\lambda\rangle$ in a wide range of imposed frequency. They have shown that there is a close similarity between the modulation characteristics of $\langle\lambda\rangle$ and those of the turbulent wall shear stress intensity. The flow visualization investigation of Brereton and Hwang (1994) is somewhat contradictory and does not agree with the probe data quoted before, in particular in the relaxation regime. More systematic studies are undoubtedly needed to clarify the reaction of the spanwise structure of the flow to imposed unsteadiness. This issue is important in the understanding of the regeneration of the coherent structures, since their genesis is strongly connected with the streaky organization of the near wall flow (Jiménéz, 1994).

The work presented here is part of a project dealing with longitudinal, normal and transverse length scales in an unsteady turbulent channel flow. The space-time spanwise correlations between the wall shear stress and longitudinal velocity fluctuations in the buffer layer are investigated here. The longitudinal length scales together with the validity of the Taylor hypothesis in these nonequilibrium flows will be published elsewhere.

The major contributions of this paper are threefold. First the difficult problem concerning the spectral characterization of the unsteady near wall turbulence is addressed in Section 3. In stationary turbulence, the autocorrelation function of fluctuating quantities depends only on the time delay. The power spectrum is one-dimensional and easily computed by standard techniques. The unsteady wall flows are nonstationary by definition. Both the autocorrelation function and the power spectrum are two-dimensional and the latter is the double Fourier transform of the first. The spectral characterization of unsteady turbulence requires a representation in the entire frequency plane bounded by the Kolmogoroff frequency $f_{K} \times f_{K}$. This is not only difficult from a technical point of view, but the interpretation of the results obtained is also much less clear than for the stationary turbulence. The periodically forced turbulent flows have, fortunately, specific properties that simplify considerably the problem. That will be discussed in some detail in this paper.

The active eddies in canonical turbulent wall flows contribute directly to the turbulence production, while the passive outer layer structures contribute only to the kinetic energy. They respectively correspond to the quasi-streamwise coherent structures in the buffer layer, and the outer structures extending to the far logarithmic layer. The characteristics of these structures may be experimentally studied through temporally filtered velocity signals (Naguib and Wark, 1992). Both active and passive eddies have well defined time scales in stationary wall turbulence. Both of them originate from the wall, but their strain rate histories are different. The inactive eddies are large-scale motions mainly associated with pressure fluctuations and they consequently scale with integral variables. The active structures are governed by local equilibrium, thus, they are universal, self-similar and they scale with the local shear stress. Whether these characteristics are still valid or not in nonstationary turbulence are unclear. The response of active and passive motions to imposed shear oscillations together with their repercussions on the correlated structure of the buffer layer constitute the second major contribution of this investigation. It is for instance asked whether the modulation characteristics, i.e., the amplitude and phase of active and inactive eddies scale respectively with the inner and outer scales. The modulation of the inner scale is directly related to that of the wall shear stress, while the outer scale is connected to the modulation of the centerline velocity. Both are considerably different when the imposed frequency is high enough. The response of outer and inner eddies is, in a first stage, determined through their contribution to the turbulent wall shear stress intensity and longitudinal shear stress. We subsequently investigate the impact of these structures to the spanwise organization of wall turbulence and show that their reactions to imposed shear oscillations are significantly different.

One of the kinetic consequences of Reynolds shear stress producing quasi-streamwise vortices in the buffer layer is the formation of the zones of low and high speed streaks that are quasi-periodical in the spanwise direction. The distance between these zones, in other words, the streak spacing, provides a well-defined spanwise scale of the canonical wall turbulence which is about 100 in wall units. The turbulence activity near the wall and the streak spacing are interconnected. For instance, there is a large consensus in the drag reduction research community that large bursting activity result in small streak spacing and vice versa. We will show here that this connection still holds in nonstationary turbulence, though with some subtle modifications. The discussion in Section 4 deals with the plausible reasons of this coincidence and is conducted in the light of recent research in the area.

\section{Definitions, experimental set-up and data reduction}

\subsection{Flow facility and hot-film measurements}

The experiments were performed in the unsteady water channel described in detail in Tardu et al. (1994). 
The sinusoidal velocity oscillations are generated by means of a specific pulsating device that allows the independent control of the mean centerline velocity, the amplitude and frequency of the imposed unsteadiness. The centerline velocity is $\bar{U}_{\mathrm{c}}=17.5 \mathrm{~cm} / \mathrm{s}$ corresponding to a Reynolds number based on the half height of the channel of $R e_{\mathrm{h}}=\frac{\bar{U}_{\mathrm{c}} h}{v}=8800$. The imposed amplitude is $20 \%$ of the centerline velocity throughout the whole study. The imposed frequency in wall units $f^{+}=f v \sqrt{u}_{\tau}^{2}$ varied by a factor 24 from $f^{+}=2.2 \times 10^{-4}$ to $f^{+}=60 \times 10^{-4}$. Hereafter ${ }^{+}$designates variables normalized by the cinematic viscosity $v$ and shear velocity $\bar{u}_{\tau}$.

The spanwise time-space correlations between the wall shear stress and the velocity at $y^{+}=10$ were performed by means of a flush-mounted TSI-1268 W hot film at the wall and a rake of four TSI F1276-10W hot films separated by $\Delta^{+}=60-80$. Spanwise and vertical probe displacements were made using a controlled traversing mechanism. The sensitive parts of the hot films are $81_{v}$ for the wall probe and $41 v$ for the hot films at $y^{+}=10$. The hot films were operated at constant temperature with 5-8\% overheat by AHARONI AN-1003 anemometer units. The calibration of the hot films was done in situ as described by Tardu et al. (1994) in which the frequency response of hot film gauges and other experimental details are also discussed. Some related details are given in Appendix A.

The analog to digital conversion was achieved with an Analog-Device RTI-800 board (accuracy 11 bit+ sign; 8 channel) installed in a PC computer. The sampling frequency was $1.5 \bar{u}_{\tau}^{2} / \nu$. The signals were filtered with accurate cut-off frequencies. The minimum record length was $T_{\mathrm{r}}=9200 h / \bar{U}_{\mathrm{c}}=191000 v \sqrt{u_{\tau}^{2}}$ for each signal which means about $45 \mathrm{~min}$ for each experiment. This give a total number of 286000 data points per probe which is large compared to 32000 points in Finnicum and Hanratty's experiments (1988) although the total duration of their data is comparable.

The classical triple decomposition is used. A quantity $q$ is decomposed into a mean $\bar{q}$ an oscillating $\tilde{q}$ and fluctuating $q^{\prime}$ components. The angle brackets designate the phase averages i.e. $\langle q\rangle=\bar{q}+\tilde{q}$. The modulation characteristics of $\langle q\rangle$ are described by the amplitude $[q]$ and phase $\{q\}$ of the fundamental mode, i.e. $\tilde{q}=[q] \cos (2 \pi f t+\{q\})$. The relative amplitude is $\hat{q}=[q] / \bar{q}$ is also introduced for convenience.

\section{Results}

\subsection{Modulation characteristics of the shear}

One of the interesting features of the unsteady flows is the coexistence of a purely viscous oscillating Stokes component with an unaltered time mean base flow when

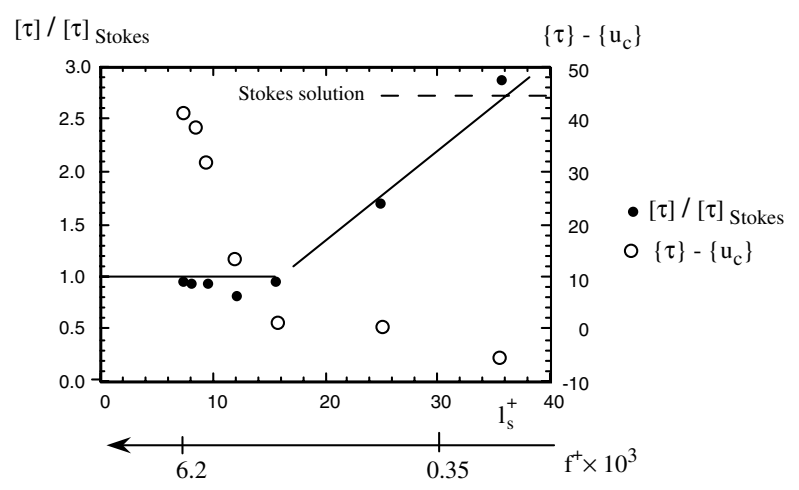

Fig. 1. Amplitude and phase shift of the wall shear stress.

the imposed frequency is larger than $f^{+}=0.003$. This observation led some research groups to use the Stokes length in wall units $l_{\mathrm{s}}^{+}=\sqrt{\frac{l}{\pi f^{+}}}$as the frequency parameter (Ronneberger and Ahrens, 1977; Tardu et al., 1994). Fig. 1 illustrates this peculiarity through the amplitude and phase shift of the wall shear stress $\langle\tau\rangle$. It is seen that when $l_{\mathrm{s}}^{+}<10,[\tau] \approx[\tau]_{\text {Stokes }}=\sqrt{2} \mu\left[u_{\mathrm{c}}\right] / l_{\mathrm{s}}$ and $\{\tau\}-\left\{u_{\mathrm{c}}\right\} \approx 45^{\circ}$. Consequently, the relative amplitude and phase shifts of the shear are fairly well described in this frequency regime by

$\frac{\partial \hat{u}}{\partial y} \approx \hat{\tau} \mathrm{e}^{-y^{+} / l_{\mathrm{s}}^{+}}=\hat{\tau} \mathrm{e}^{-y^{+} \sqrt{\pi f^{+}}}$

$\left\{\frac{\partial u}{\partial y}\right\}-\left\{u_{\mathrm{c}}\right\} \approx \frac{\pi}{4}-\frac{y^{+}}{l_{\mathrm{s}}^{+}} \quad$ (in radians)

The oscillating shear is therefore mainly confined in $y^{+}<2 l_{\mathrm{s}}^{+}$. In the quasi-steady limit, in return, $\langle\tau\rangle_{\mathrm{qs}} \propto\left\langle U_{\mathrm{c}}\right\rangle^{2}$ which gives $\hat{\tau}_{\mathrm{qs}} \approx 2 \hat{u}_{\mathrm{c}}$ and $\left\{\tau_{\mathrm{qs}}\right\} \approx\left\{u_{\mathrm{c}}\right\}$ for small amplitudes, where "qs" refers to the quasi-steady regime hereafter.

\subsection{Modulation of the turbulent wall shear stress intensity and the turbulent intensity at $y^{+}=10$}

Fig. 2a shows the relative amplitude of the wall shear stress intensity $\left\langle\tau^{\prime} \tau^{\prime}\right\rangle$ related to the relative amplitude of the wall shear stress $\langle\tau\rangle$ versus the imposed frequency $f^{+}$. The corresponding ratio $\widehat{u^{\prime} u^{\prime}} / \hat{u}$ of the $\left\langle u^{\prime} u^{\prime}\right\rangle$ modulation and that of the local velocity $\langle u\rangle$ at $y^{+}=10$ is also shown in Fig. 2a. Note first that the measurements in the low frequency regime agree well with the expected quasisteady behaviors. In the low frequency range, one has $\frac{\left\langle\tau^{\prime} \tau^{\prime}\right\rangle_{\mathrm{qs}}}{\langle\tau\rangle_{\mathrm{qs}}^{2}}=0.38^{2}$ and a Taylor series analysis of this relationship leads to $\widehat{\tau^{\prime} \tau^{\prime}}{ }_{\mathrm{qs}} \approx 2 \hat{\tau}_{\mathrm{qs}}$. This is well born out with the measurements at $f^{+}=0.0002$ shown in Fig. 2a. Similar arguments apply to $\left\langle u^{\prime} u^{\prime}\right\rangle$ in the low imposed frequency regime (Tardu et al., 1994).

The modulations of both $\left\langle\tau^{\prime} \tau^{\prime}\right\rangle$ and $\left\langle u^{\prime} u^{\prime}\right\rangle$ decrease when the imposed frequency increases but the decay 
$\widehat{\tau^{\prime} \tau^{\prime}} / \hat{\tau}$ and $\widehat{u^{\prime} u^{\prime} /} \widehat{u}$
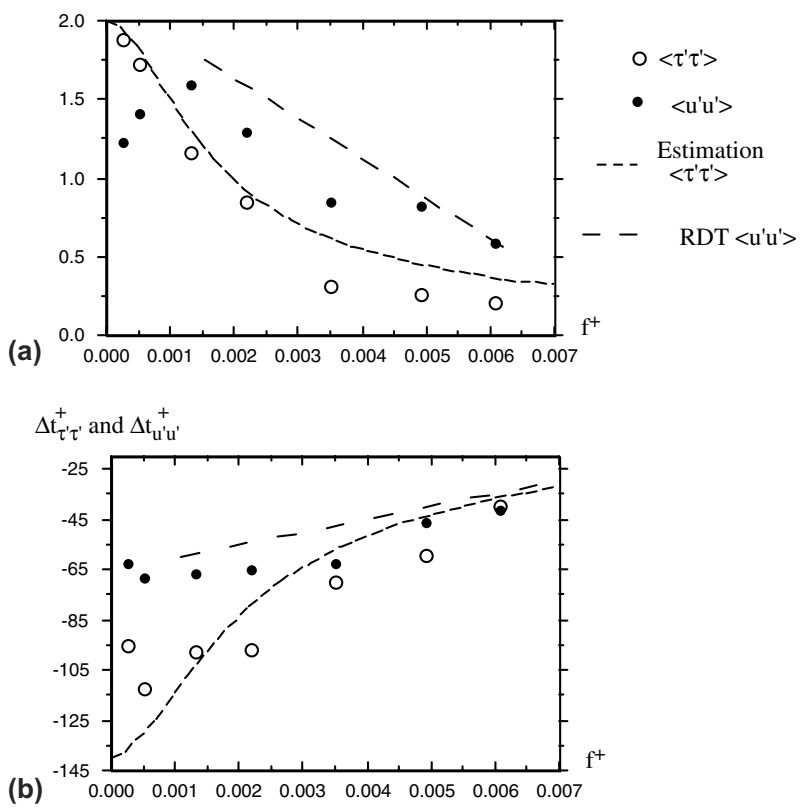

Fig. 2. Modulation of the turbulent intensity at $y^{+}=10$ and of the wall shear stress intensity vs. the imposed frequency. Comparison with a first order model and RDT from Brereton and Mankbadi (1993): (a) ratio of relative amplitudes; (b) time lags.

$\widehat{\tau^{\prime} \tau^{\prime}} / \hat{\tau}$ in is more pronounced and takes place earlier. These results are in good agreement with previously published data. The weakening of the modulation of the turbulence in this frequency regime, may be explained by the fact that the near wall flow responds only partly to the rapid temporal changes of the shear $\frac{\partial \tilde{u}}{\partial y}$. Suppose that the wall sees an effective shear $\frac{\partial \tilde{u}_{\text {eff }}^{+}}{\partial y^{+}}$which may be modeled through a first order time-response equation $\theta^{+} \frac{\partial}{\partial t^{+}}\left[\frac{\partial \tilde{u}_{\text {eff }}}{\partial y^{+}}\right]+\frac{\partial \tilde{u}_{\text {fff }}^{+}}{\partial y^{+}}=\frac{\partial \tilde{u}^{+}}{\partial y^{+}}$where $\theta^{+}$stands for the relaxation time in wall units. This introduces a damping ratio $\frac{1}{\sqrt{1+\left(2 \pi f^{+} \theta^{+}\right)^{2}}}$ and a phase shift $-\operatorname{arctg}\left(2 \pi f^{+} \theta^{+}\right)$between the modulation of the real and the effective shears. This crude model results in sharp decreases of the amplitudes, and constant time lags $\Delta t_{\tau^{\prime} \tau^{\prime}}^{+}=\left\{\tau^{\prime} \tau^{\prime}\right\}-\{\tau\} / 2 \pi f^{+} \approx-\theta^{+}$ in the quasi-steady regime, and $\Delta t_{\tau^{\prime} \tau^{\prime}}^{+} \approx-\frac{1}{4 f^{+}}$when the imposed frequency is sufficiently high. The resulting profiles shown by the dotted lines in Fig. $2 a$ and $b$ have been obtained through best curve fitting to give $\theta^{+}=140$ at the wall. It is seen that this first order model estimates reasonably well the amplitude and the time lag of $\left\langle\tau^{\prime} \tau^{\prime}\right\rangle$. The modulation characteristics of $\left\langle u^{\prime} u^{\prime}\right\rangle$ at $y^{+}=10$ interpolated from an unsteady closure based on the rapid-distortion theory (RDT) are also shown in Fig. 2. This model uses the ratio of the turbulent shear stress to the turbulent kinetic energy to be a function of effective strain and of the distortion story of the turbulent field (Maxey, 1982). The details are beyond the scope of this paper and may be found in Mankbadi and
Liu (1992) and Tardu and Da Costa (2001). It is seen that the RDT predicts reasonably well the behavior of $\left\langle u^{\prime} u^{\prime}\right\rangle$, except a slight underestimation of $\Delta t_{u^{\prime} u^{\prime}}^{+}=\left\{u^{\prime} u^{\prime}\right\}-$ $\{u\} / 2 \pi f^{+}$in the low frequency regime.

\subsection{Unsteady spanwise correlation coefficient between the wall shear stress and the streamwise velocity}

The response of the near wall structures in the spanwise direction is investigated by performing the phase locked ensemble averages of the correlations in time and space:

$$
\langle R\rangle=\frac{\left\langle\tau^{\prime}\left(x^{+}=0, z^{+}=0, t\right) u^{\prime}\left(x^{+}=0, y^{+}=10, z^{+}, t+\Delta t\right\rangle\right.}{\sqrt{\left\langle\tau^{\prime} \tau^{\prime}\right\rangle} \sqrt{\left\langle u^{\prime} u^{\prime}\right\rangle}}
$$

between the fluctuating wall shear stress $\tau^{\prime}(t)$ and the streamwise velocity $u^{\prime}(t)$ at $y+=10$. The time mean correlation coefficient $\overline{\langle R\rangle}$, the amplitude $[R]$ and the phase $\langle R\rangle$ of the fundamental Fourier mode are subsequently determined as alluded earlier. The time delay is $\Delta t=0$ through the whole study.

The profiles of the unsteady time-mean spanwise correlation coefficient $\overline{\langle R\rangle}$ are shown in Fig. 3. They are compared with the $\bar{R}$ distribution measured in situ in steady flow. The collapse of the profiles for different imposed frequencies indicates that $\langle R\rangle$ is unaffected at the mean by the imposed unsteadiness. The results of Naguib and Wark (1992, NW hereafter) obtained in a standard boundary layer at $R e_{\theta}=1579$ are also shown in Fig. 3 for comparison. There is a nice concordance between the data of these authors and the results discussed here, despite the differences in $\operatorname{Re}_{\theta}$.

The modulation characteristics of the correlation coefficient at several spanwise offsets are summarized in Fig. 4 versus the imposed frequency. Both the amplitude and phase of $\langle R\rangle$ are related to their quasi-steady values. This representation takes into account the large variations of $[R]$ and $\{R\}$ via $z^{+}$to a certain degree, and the data is fairly well regrouped especially for the phase shifts (Fig. 4b). The quasi-steady amplitude $\left[R_{\mathrm{qs}}\right]$ and phase $\left\{R_{\mathrm{qs}}\right\}$ are independently determined by careful

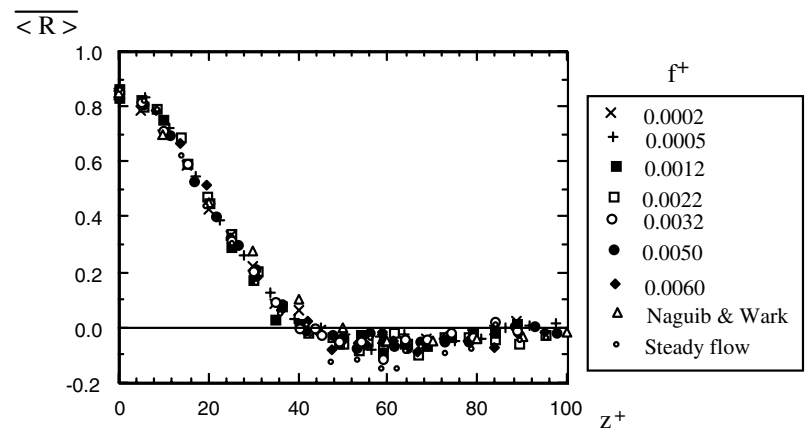

Fig. 3. Distribution of the time mean correlation coefficient. 


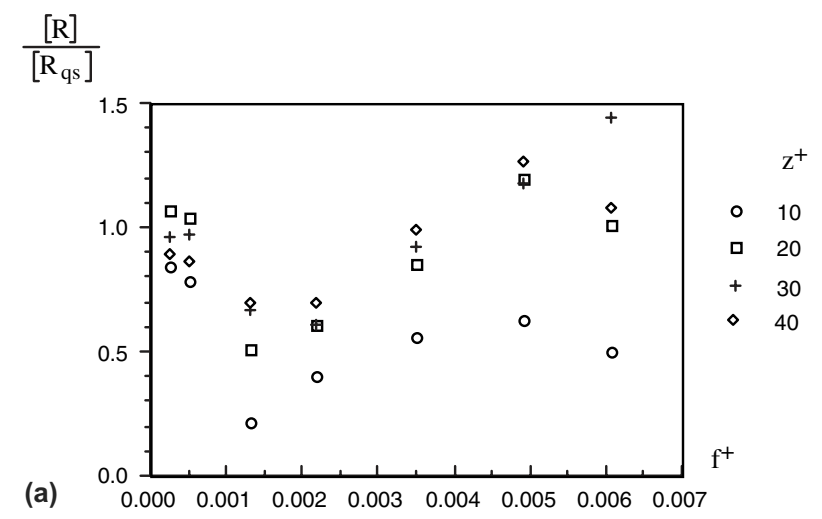

$\Delta \mathrm{t}_{\mathrm{R}}^{+}=\frac{\{\mathrm{R}\}-\left\{\mathrm{R}_{\mathrm{qs}}\right\}}{2 \pi \mathrm{f}^{+}}$

(b)

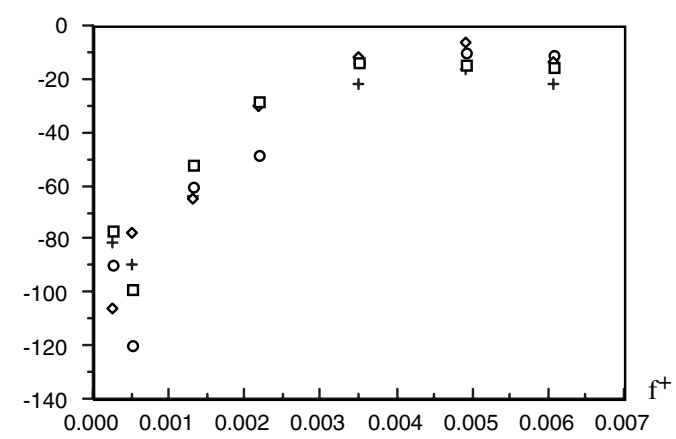

Fig. 4. Modulation characteristics of the correlation coefficient: (a) amplitude versus the imposed frequency at four typical spanwise locations; (b) time lag.

measurements at $f^{+} \rightarrow 10$. These details are of minor importance and they may be found in Vezin et al. (1994).

The scaled amplitude $[R] /\left[R_{\mathrm{qs}}\right]$ decreases in the frequency range $f^{+}<0.0022$, as a result of the finite response time of the near wall structures. This decay persists in the entire range $z^{+}<50$, where the time mean correlation coefficient is positive and relatively large. There is a change in the reaction of the correlated buffer layer structures at larger forcing frequencies. The amplitude $[R]$ increases with $f^{+}>0.0022$, resulting in large cyclic variations of the intrinsic properties of the near wall turbulence in the high frequency regime.

The time lag of the correlation coefficient defined as $\Delta t_{R}^{+}=\frac{\langle R\rangle-\left\langle R_{\mathrm{qs}}\right\rangle}{2 \pi f^{+}}$is shown in Fig. 4b. It is seen that $\Delta t_{R}^{+} \approx-100$ in the low frequency range, decreases by a factor 3 at $f^{+}=0.0022$ and reaches a plateau region at larger $f^{+}$. This behavior is consistent with the recovery of the correlation coefficient response and cannot be predicted by a first order relaxation model. It is noteworthy that these structural modifications occur when $f^{+}$is larger than the critical frequency $f_{\mathrm{cr}}^{+}=0.0022$, and thus, when the oscillating shear is mainly confined in the low buffer layer. The repercussion of the inner active and the outer passive eddies on these peculiar aspects of the near wall response is addressed in the next section.
3.4. Investigation of the spanwise correlation coefficient by temporal filtering

\subsubsection{Spectral characterization of unsteady flows with imposed time periodicity}

The statistical quantities related to turbulence in unsteady forced flows are time periodical. Such processes are called cyclostationary in wide sense with period $T$, in information theory (CSP(T) hereafter). The CSP(T) are encountered not only in unsteady turbulent channel flows, but also in turbulent boundary layers subjected to localized unsteady blowing/suction to control the drag, or the forced synthetic jets used in bluff bodies separation control, pulsating jets, etc. The time periodical random processes are studied in details in information theory since three decades (see Gardner and Franks, 1975, for an excellent review). Our community is not entirely aware of these investigations. The aim of this session is to give a concise resume on the cyclostationary processes based essentially on Hurd (1989), see also Tardu, 2003). We also connect the CSP(T) characteristics to the classical phase averaging process.

3.4.1.1. Definitions. Let $x(t)$ be a continuous second-order random function and let the correlation function:

$R_{x}\left(t_{1}, t_{2}\right)=E\left[x\left(t_{1}\right) x^{*}\left(t_{2}\right)\right]$

with $E$ denoting the statistical expectation operator and the superscript ${ }^{*}$ standing for complex conjugation. One has

$R_{x}\left(t_{1}, t_{2}\right)=\int_{-\infty}^{\infty} \int_{-\infty}^{\infty} \exp \left[2 \pi j\left(t_{1} f_{1}-t_{2} f_{2}\right)\right] r_{Y}\left(\mathrm{~d} f_{1}, \mathrm{~d} f_{2}\right)$

where $r_{Y}\left(\mathrm{~d} f_{1}, \mathrm{~d} f_{2}\right)$ represents the part of the frequency domain $f_{1} \times f_{2}$ wherein the energy is concentrated (see, Hurd, 1989 p. 57 for a rigorous definition). The stochastic process $x(t)$ is wide sense cyclostationary of periodicity $T$ (or periodically correlated) when

$R_{x}\left(t_{1}, t_{2}\right)=E\left[x\left(t_{1}\right) x^{*}\left(t_{2}\right)\right]=R_{x}\left(t_{1}+m T, t_{2}+n T\right)$

where $m$ and $n$ are any integer and $E$ stands for expected value. Periodically correlated processes include wide sense stationary processes, for which $R_{x}\left(t_{1}, t_{2}\right)=$ $R_{x}\left(t_{2}-t_{1}\right)$ for every $t_{1}, t_{2}$.

3.4.1.2. Characterization of cyclostationary processes. The cyclostationarity expressed through (3) implies

$R_{x}\left(t_{1}, t_{2}\right)=\frac{1}{2 N+1} \sum_{k=-N}^{k=N} R_{x}\left(t_{1}+k T, t_{2}+k T\right)$

Considering a harmonizable process $x(t)$, the relationship (2) combined with (4) leads to

$$
\begin{aligned}
R_{x}\left(t_{1}, t_{2}\right)= & \frac{1}{2 N+1} \sum_{k=-N}^{k=N} \int_{-\infty}^{\infty} \int_{-\infty}^{\infty} \exp \left[2 \pi j\left(t_{1}+k T\right) f_{1}\right. \\
& \left.-2 \pi j\left(t_{2}+k T\right) f_{2}\right] r_{Y}\left(\mathrm{~d} f_{1}, \mathrm{~d} f_{2}\right)
\end{aligned}
$$


and after some algebra to

$$
\begin{aligned}
R_{x}\left(t_{1}, t_{2}\right)= & \int_{-\infty}^{\infty} \int_{-\infty}^{\infty} \exp \left[2 \pi j\left(t_{1} f_{1}-t_{2} f_{2}\right)\right] \\
& \times D_{N}\left(f_{1}, f_{2}\right) r_{Y}\left(\mathrm{~d} f_{1}, \mathrm{~d} f_{2}\right)
\end{aligned}
$$

where, $D_{N}$ equals to

$$
\begin{aligned}
D_{N}\left(f_{1}, f_{2}\right) & =\frac{1}{2 N+1} \sum_{k=-N}^{k=N} \exp \left[2 \pi j k T\left(f_{1}-f_{2}\right)\right] \\
& =\frac{1}{2 N+1}\left\{1+2 \sum_{k=1}^{k=N} \cos \left[2 \pi k T\left(f_{1}-f_{2}\right)\right]\right\}
\end{aligned}
$$

Eqs. (1) and (5) are identical. Thus, $D_{N}$ has to be equal to one. According to (6), this is possible if and only if

$f_{2}=f_{1}-\frac{j}{T}$

$j$ standing for any integer. Thus, the support of a periodically correlated process of period $T$, is contained in a set of equally spaced lines parallel to the main diagonal. This is schematically shown in the frequency plane $f_{1} \times f_{2}$ in Fig. 5. The lines $f_{2}=f_{1}-\frac{j}{T}$ are randomly distributed, the distribution depending on the unsteady process itself.

The support of the time mean correlation is the diagonal $f_{2}=f_{1}$. To show this, first write (1) as

$$
\begin{aligned}
R_{x}\left(t_{1}, t_{1}+t\right)= & \int_{-\infty}^{\infty} \int_{-\infty}^{\infty} \exp \left[2 \pi j\left[t_{1}\left(f_{1}-f_{2}\right)-t f_{2}\right]\right] \\
& \times r_{Y}\left(\mathrm{~d} f_{1}, \mathrm{~d} f_{2}\right)
\end{aligned}
$$

where $t=t_{2}-t_{1}$. The time mean $\bar{R}_{x}$ is by definition

$$
\begin{aligned}
\bar{R}_{x}\left(t_{1}, t\right)= & \lim _{\Gamma \rightarrow \infty} \frac{1}{2 \Gamma} \int_{t_{1}-\Gamma}^{t_{1}+\Gamma} R_{x}\left(t_{1}, t_{1}+t\right) \mathrm{d} t_{1} \\
= & \lim _{\Gamma \rightarrow \infty} \frac{1}{2 \Gamma} \int_{-\infty}^{\infty} \int_{-\infty}^{\infty} \exp \left(-2 \pi j t f_{2}\right) r_{Y}\left(\mathrm{~d} f_{1}, \mathrm{~d} f_{2}\right) \\
& \times \int_{t_{1}-\Gamma}^{t_{1}+\Gamma} \exp \left[2 \pi j t_{1}\left(f_{1}-f_{2}\right)\right] \mathrm{d} t_{1}
\end{aligned}
$$

Computing the last integral leads to

$$
\begin{aligned}
\bar{R}_{x}\left(t_{1}, t\right)= & \lim _{\Gamma \rightarrow \infty} \int_{-\infty}^{\infty} \int_{-\infty}^{\infty} \exp \left[2 \pi j\left[t_{1}\left(f_{1}-f_{2}\right)-t f_{2}\right]\right] \\
& \times \frac{\sin \left[2 \pi\left(f_{1}-f_{2}\right) \Gamma\right]}{2 \pi\left(f_{1}-f_{2}\right) \Gamma} r_{Y}\left(\mathrm{~d} f_{1}, \mathrm{~d} f_{2}\right)
\end{aligned}
$$

The limit of the integral in the right hand side is zero every where except on the diagonal $f_{1}=f_{2}$. That results in

$\bar{R}_{x}\left(t_{1}, t\right)=\iint_{f_{1}=f_{2}} \exp \left(-2 \pi j t f_{2}\right) r_{Y}\left(\mathrm{~d} f_{1}, \mathrm{~d} f_{2}\right)=\bar{R}_{x}(t)$

and the time mean is stationary of course, since $\bar{R}_{x}$ depends only on the delay $t=t_{2}-t_{1}$. The support of the modulation $\widetilde{R}_{x}$ is the ensemble of the lines $f_{2}=f_{1}-\frac{j}{T}$, with $j \neq 0$, the diagonal being excluded. The following remarks are important for the characterization and interpretation of unsteady turbulent wall flows:

1. Fig. 5 shows that the frequency domain of a low pass filter of cut off frequency smaller than half of the imposed frequency $\Delta f_{\mathrm{LP}} \leqslant \frac{1}{2 T}$ intersects only the diagonal $f_{1}=f_{2}$. Thus the output of such a filter will be strictly stationary. Similarly, a $\operatorname{CSP}(\mathrm{T})$ when passed

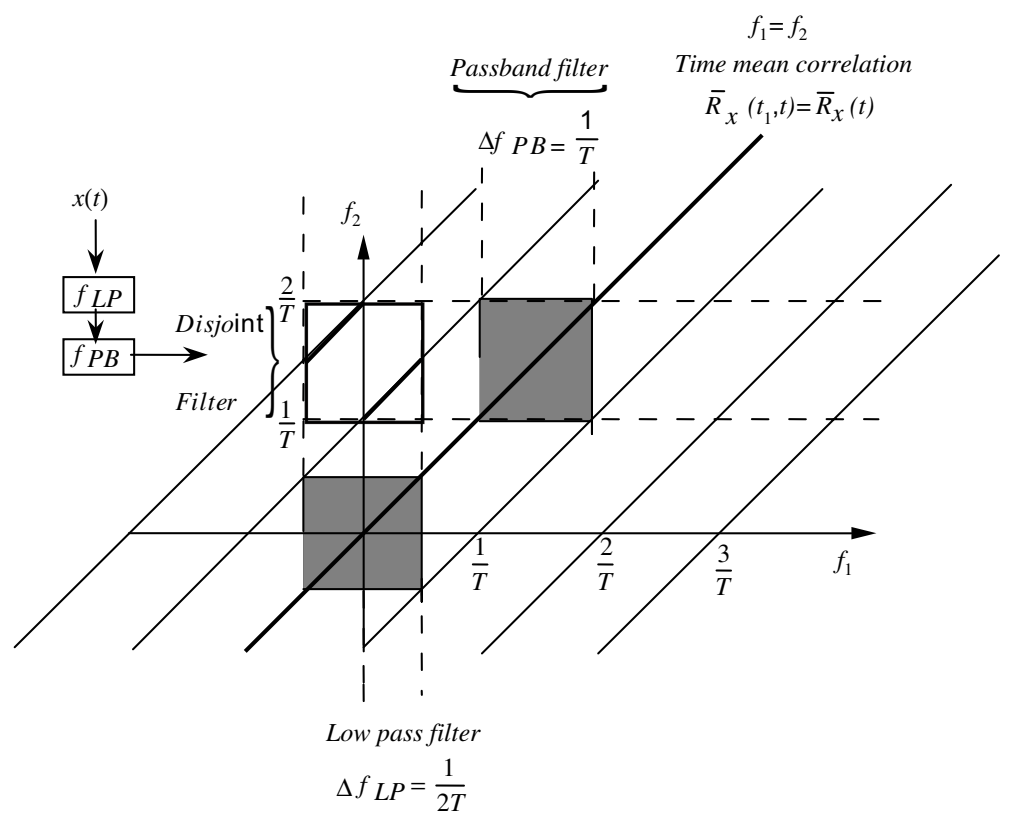

Fig. 5. Support of a cyclostationary process in the frequency plane. 
through a band pass filter with band pass $\Delta f_{\mathrm{BP}} \leqslant \frac{1}{T}$ is also stationarized and its statistics have strictly zero modulation. In this study, we proceeded by temporal filtering to investigate the response of large and smallscale structures, as it will be discussed in the following. Specific care has to be taken in choosing the characteristics of the temporal filters for unsteady forced periodical turbulent flows. If, for example, a low pass filter with cut off frequency smaller than $\frac{1}{2 T}$ is chosen for the fluctuating longitudinal velocity $u^{\prime}$ the modulation of the output intensity is ineluctably $\widetilde{u_{0}^{\prime} u_{0}^{\prime}}=0$, not because the outer scales are frozen and cannot follow the imposed unsteadiness, but simply because the process is cyclostationary.

2. The output of a disjoint filter is not null when the input is a $\operatorname{CSP}(\mathrm{T})$ contrarily to a stationary process. Disjoint filtering consists of passing the signal through two filters whose bandwidths do not intersect. Such an operation is shown schematically in Fig. 5. The signal is first low pass filtered and passes consequently through a band pass filter defined on the interval $\rfloor \frac{1}{T}, \frac{1}{2 T}\lfloor$. The frequency domain covered by this operation contains the segments of the lines $f_{2}=f_{1}+\frac{1}{T}$ and $f_{2}=f_{1}+\frac{2}{T}$ that are part of the of the frequency support. Thus, the time means statistics of the output are strictly zero, but not their modulation. Such interactions between disjoint scales have to be taken into account in unsteady forced flows.

\subsubsection{Choice of invariant time filters}

The band-pass filtered wall shear stress and streamwise velocity fluctuations were further analyzed. The choice of the filters resulted from a compromise between three factors. First, the filters have to recover the unsteady support as discussed in the previous session. The imposed frequency is varied in a large range in this investigation, but the bandwidths are kept constant to insure consistency from one case to another. The third factor is the connection between this study and published data for confrontation purposes. There is, indeed, at least one set of data in steady, canonical near wall turbulence (NW) and that will allow us to compare the time mean statistics. Thus, three digital, zero phaseshift, 128 points FIR (finite impulse response) filters have been designed. They are designated as filter 0 (lowpass, cut-off frequency $\Delta f_{0}^{+}=0-0.0045$ ), filter 1(bandpass $\Delta f_{1}^{+}=0.0055-0.022$ ) and filter 2 (band-pass $\left.\Delta f_{2}^{+}=0.0316-0.0482\right)$. The filtered instantaneous flow quantities are denoted by $q_{i}^{\prime}(t)$, where $i=0-2$ stands for the output of the filters $0-2$.

All of the three filters recover the unsteady support in the imposed frequency range investigated here. Filters 1 and 2 are exactly the same as in NW. The cut-off frequency of the low pass filter used by NW is 0.0025 . It has to be increased to 0.0045 to recover the unsteady support at the two highest imposed frequencies. This modification did not appreciably affect the modulation characteristics of the low pass filtered signals, in the low and medium imposed frequency ranges.

\subsubsection{Interaction powers}

The interaction powers related to the disjoint parts of the spectral domain are not necessarily zero, contrarily to the stationary processes, which admit spectral decomposition. We discussed this point previously in Section 3.4.1. For example, $\left\langle u_{0}^{\prime} u_{1}^{\prime}\right\rangle$ may be strongly modulated, though $\overline{u_{0}^{\prime} u_{1}^{\prime}}=0$ at the mean. It is not excluded that the phase averaged interaction power $\left\langle u_{0}^{\prime} u_{1}^{\prime}\right\rangle$ reaches cyclic values larger than $\left\langle u_{0}^{\prime} u_{0}^{\prime}\right\rangle$ or $\left\langle u_{1}^{\prime} u_{1}^{\prime}\right\rangle$. If that happens, the analysis cannot be limited only to the behavior of the pass-band powers $\left\langle u_{i}^{\prime} u_{i}^{\prime}\right\rangle$. It should include the response of the interaction powers too. The largest value of $\left\langle u_{0}^{\prime} u_{1}^{\prime}\right\rangle$ during the oscillation cycle is $I_{0,1} \approx\left[u_{0}^{\prime} u_{1}^{\prime}\right]$. It is compared with the band pass power $I_{0,0} \approx \overline{u_{0}^{\prime} u_{0}^{\prime}}+\left[u_{0}^{\prime} u_{0}^{\prime}\right]$ in Fig. 6 , rather than $I_{1,1}$ which is two times larger. The distributions $\frac{I_{0,2}}{I_{2,2}}$ and $\frac{I_{1,2}}{I_{2,2}}$ determined in the same way are also summarized in Fig. 6 versus the imposed frequency. It is seen that the modulation of the interaction powers between structures of large-scale differences, such as $\left\langle u_{0}^{\prime} u_{2}^{\prime}\right\rangle$ is negligible. However, the interactions between "adjacent" structures as $\left\langle u_{0}^{\prime} u_{1}^{\prime}\right\rangle$ and $\left\langle u_{1}^{\prime} u_{2}^{\prime}\right\rangle$ become somewhat significant once $f^{+}>0.004$. Their contributions do not exceed $20 \%$, so that the passband powers are still predominant. Yet, a net trend of increase is noticeable, and it is plausible that the interaction powers dominate the dynamics at further higher imposed frequencies.

\subsubsection{Modulation characteristics of the filtered fluctuat-} ing wall shear stress and fluctuating streamwise velocity

The contributions in unsteady flow of the filters 1,2 and 3 to the turbulence intensity at $y^{+}=10$ are respectively $0.27,0.45$ and 0.1 with a total of $85 \%$. Note that the contribution of the outer structures is half that of the near wall eddies. These values are in good agreement with Naguib and Wark (1992). Similar results

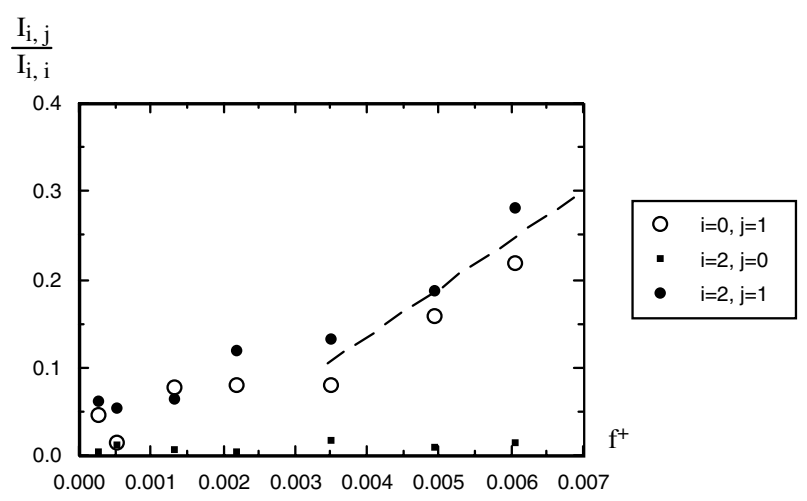

Fig. 6. Maximum of the phase averages of the interaction powers. 
have been obtained for the fluctuating wall shear stress, with only some subtle differences.

The relative amplitudes of the band pass filtered signals are summarized in Fig. 7. The frequency dependence of $\overline{u_{i}^{\prime} u_{i}^{\prime}}$ and $\overline{\tau_{i}^{\prime} \tau_{i}^{\prime}}$ are compared with that of the unfiltered signals. It is seen that, the cyclic contributions of the inner and outer structures feature on the whole the same qualitative and quantitative behaviors. They decrease from the quasi-steady limit with more or less the same frequency sensitivity in the relaxation regime. Some subtle differences in the reaction of the inner eddies are however perceptible at $f^{+}>0.004$. Note that $\tau_{2}^{\prime} \tau_{2}^{\prime}$ exhibits a net tendency to increase with $f^{+}$(Fig. $7 \mathrm{~b})$. Apart from these exceptions, and referring to the global trend of the relative amplitudes, one would conclude that the near wall turbulence responds almost uniformly in the part of the spectrum scanned by the three filters. Any conclusion of this kind would be somewhat premature, without analyzing the phase shifts and the related time lags.

As a matter of fact, the time lags $\Delta t_{i, u^{\prime} u^{\prime}}^{+}$and $\Delta t_{i, \tau^{\prime} \tau^{\prime}}^{+}$ shown in Fig. 8 give evidence of important differences in the reaction of inner and outer eddies. First thing to note from this figure is the constancy of the time lags at imposed frequencies smaller than roughly $f_{\mathrm{cr}}^{+}$. Note that this critical value is nearly the cut-off frequency of filter 0 . When $f^{+}>f_{\mathrm{cr}}^{+}$, however, the time lags of the inner structures decrease uniformly and reach a plateau region further on. In return, $\Delta t_{0, u^{\prime} u^{\prime}}^{+}$and $\Delta t_{0, \tau^{\prime} \tau^{\prime}}^{+}$corresponding to the outer passive structures are insensitive to the imposed
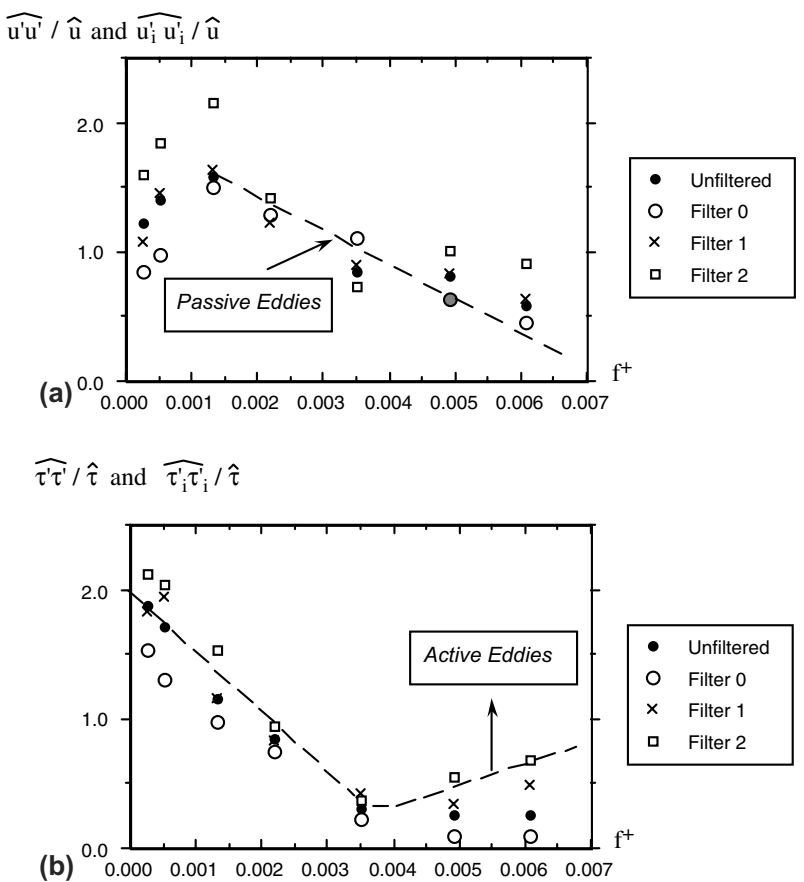

Fig. 7. Relative amplitude of the modulation of the intensity of the filtered and unfiltered signals: (a) turbulent intensity at $y^{+}=10$; (b) wall shear stress intensity.
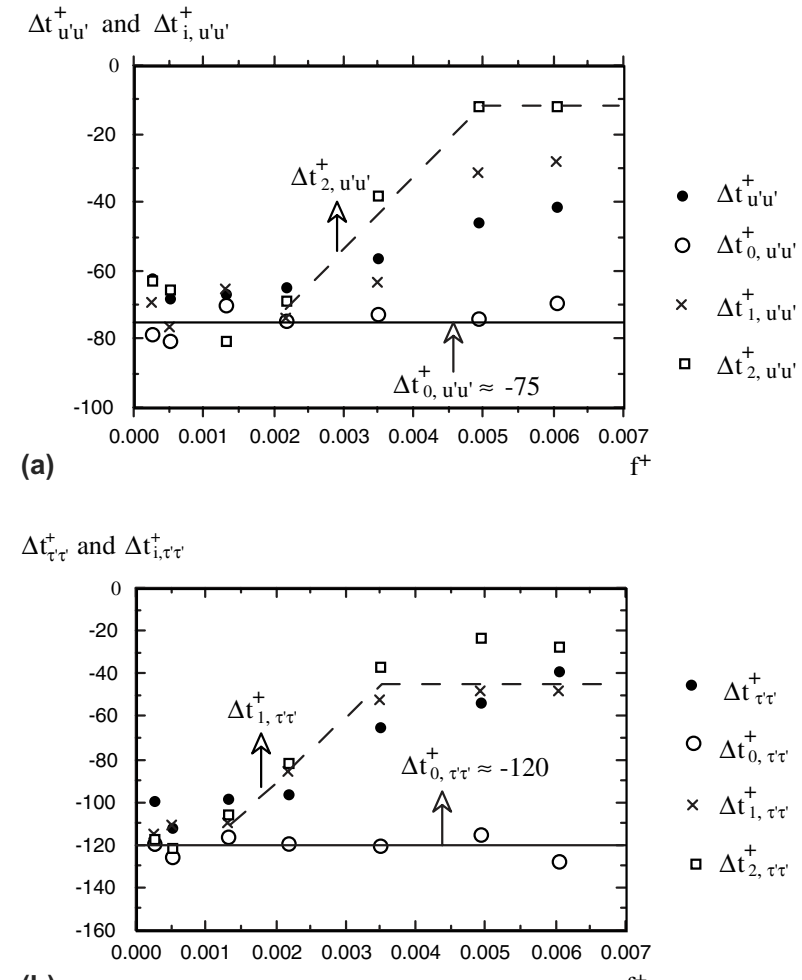

(b)

Fig. 8. Time lags of the modulation of the intensity of the filtered and unfiltered signals: (a) $\Delta t_{i, u^{\prime} u^{\prime}}^{+}=\left\{u_{i}^{\prime} u_{i}^{\prime}\right\}-\{u\} / 2 \pi f^{+}$at $y^{+}=10$; (b) $\Delta t_{i, \tau^{\prime} \tau^{\prime}}^{+}=\left\{\tau_{i}^{\prime} \tau_{i}^{\prime}\right\}-\{\tau\} / 2 \pi f^{+}$.

unsteadiness and remain remarkably close to $\Delta t_{0, u^{\prime} u^{\prime}}^{+} \approx$ -75 and $\Delta t_{0, \tau^{\prime} \tau^{\prime}}^{+} \approx-120$.

It was shown in Section 3.2 that a first order relaxation model reflects reasonably well the modulation characteristics of the near wall turbulence. Such a model is undoubtedly not adequate with regard to the structures of specific time scales. The reader will agree that the constancy of the time lags $\Delta t_{1}^{+}, \Delta t_{2}^{+}$at large frequencies $f^{+}>0.0035$, and that of $\Delta t_{0}^{+}$in the entire regime are incompatible with a first order linear minimum phase model. Nothing would imply, indeed, that the structures 0,1 or 2 are governed by linear transfer functions. The inactive motions of large scale, do not contribute to the Reynolds shear stress and do not directly produce or dissipate any significant amount of energy. They may however act indirectly by creating local regions of intense shear in the wall region and triggering local instabilities, contributing therefore, to the generation of energetic eddies. According to Bradshaw (1967), the passive structures are merely governed by pressure-strain correlations and turbulent diffusion, i.e. one has $\frac{\partial u_{0}^{\prime} u_{0}^{\prime}}{\partial t} \approx-\frac{\partial}{\partial y} \widetilde{u_{0}^{\prime 2} v_{0}^{\prime}}+\frac{2}{\rho} \widetilde{p_{0}^{\prime} \frac{\partial u_{0}^{\prime}}{\partial x}}$. The turbulent diffusion term at the right hand of this equation is nonlinear and it is neglected in the rapid distortion theory. The use of models inferred from RDT may 
therefore be questioned at least for the outer structures. The structures 1 , on the other hand, contribute mostly to the Re shear stress as clearly shown by NW. All of the terms involving $\frac{\partial\left\langle u_{u}^{\prime} u_{1}^{\prime}\right\rangle}{\partial t}$ have to be retained and the corresponding production term may directly be replaced by $-2\left\langle u^{\prime} v^{\prime}\right\rangle \frac{\partial\langle u\rangle}{\partial y}$ for a first approximation. Note, finally that the modulation characteristics of the structures 1 and 2 are basically identical. This is expected, since both structures are active, and they should scale with the modulation of the local shear stress and the distance to the wall.

Beykirch et al. (1996) investigated the frequency response of the near wall turbulence subject to imposed shear oscillations through the Wigner-Ville spectrum. The latter introduces the concept of "instantaneous frequency" $\eta$ (Flandrin, 1998), and reads for

$W_{x}(t, \eta)=\int_{-\infty}^{+\infty} \Phi_{x}\left(\eta-\frac{\zeta}{2}, \eta+\frac{\zeta}{2}\right) \exp (-j 2 \pi \zeta t) \mathrm{d} \zeta$

where $\Phi_{x}\left(f_{1}, f_{2}\right)$ is the spectra of the nonstationary signal $x(t)$. The use of Wigner-Ville spectrum in unsteady wall turbulence is not common. We derive in Appendix B the Wigner-Ville spectrum of a cyclostationary process and show that $W_{x}(t, \eta)$ is the sum:

$W_{x}(t, \eta)=\sum_{i \in Z} W_{x i}(t, \eta)$

of the discrete components $W_{x i}(t, \eta)=\Phi_{x}\left(\eta-\frac{i}{2 T}, \eta+\frac{i}{2 T}\right)$ $\exp \left(-j 2 \pi \frac{i t}{T}\right)$. For a given frequency $\eta$, the Wigner-Ville spectrum covers only partly the spectral domain delimited by a band pass filter. Fig. 18 shows this domain for an octave pass-band filter $[f, 2 f]$ when $\eta=\frac{3}{2} f$. We investigate here the phase average of the filtered signal, which, in terms of Wigner-Ville spectrum equals:

$\langle x x\rangle=\int_{\eta=f}^{\eta=2 f} W_{x}(t, \eta) \mathrm{d} \eta$

The last relationship highlights the difference between these results and the Beykirch et al.'s (1996) investigation. These authors noticed that both the real and imaginary parts of $W_{x}(t, \eta)$ vanish in the high-imposed frequency regime for octave band pass filtered signals with $\eta^{+} \approx 0.03$. We observe a decrease of amplitudes under similar conditions, but not entirely frozen turbulence response. It is, of course, not impossible that $W_{x}(t, \eta)$ vanishes, at a given "instantaneous" frequency, but not its integral. This may be the case for instance, when the unsteadiness does not penetrate into the high frequency range of the spectrum. Such processes are called weakly cyclostationary in information theory. The bold lines in Fig. 18 show the spectral support of such a signal. It is seen that $\langle x x\rangle$ is modulated in the band covered by the filter, but that the modulation $\widetilde{W}_{x}(t, \eta)$ is zero at $\eta=\frac{3}{2} f$.
3.4.5. Modulation characteristics of the spanwise correlation coefficient inferred from temporal filtering

The profiles of the time-mean correlation coefficients inferred from temporal filtering are shown in Fig. 9a and b which recapitulate respectively the distributions of $\bar{R}_{0}$ and $\bar{R}_{2}$. The "negative dip" in $\bar{R}$, appears clearly in Fig. $9 \mathrm{~b}$. It is related to the signature of the low-speed streaks and may be identified as the half streak spacing $\bar{\lambda}^{+} / 2$. One may, therefore conclude from Fig. $9 \mathrm{~b}$ that $\bar{\lambda}^{+} \approx 80$ 100 as in steady flow. Recall that such a negative local minimum is hardly distinguishable in the profiles of the conventional correlation coefficient $\bar{R}$ (Fig. 3). It is also totally absent in the $\bar{R}_{0}$ distributions (Fig. 9a). This confirms the analysis of NW, who argued that the outer structures contaminate $\bar{R}$ by tangling the footprints of the low speed streaks.

The amplitude of the correlation coefficient $\left\langle R_{0}\right\rangle$ is shown in Fig. 10. It is compared with its corresponding "quasi-steady" distribution. Notwithstanding the experimental uncertainties, the salient feature of the profiles $\left[R_{0}\right]$ is their remarkable insensitivity to the imposed frequency. Therefore, the outer layer structures keep up the same correlated reaction to the forcing in the high as well the low imposed frequency ranges. The insensitivity of $\left\langle R_{0}\right\rangle$ may be better appreciated when $\left[R_{0}\right]$ is compared with $\left[R_{1}\right]$ or $\left[R_{2}\right]$. Fig. 11 shows for instance

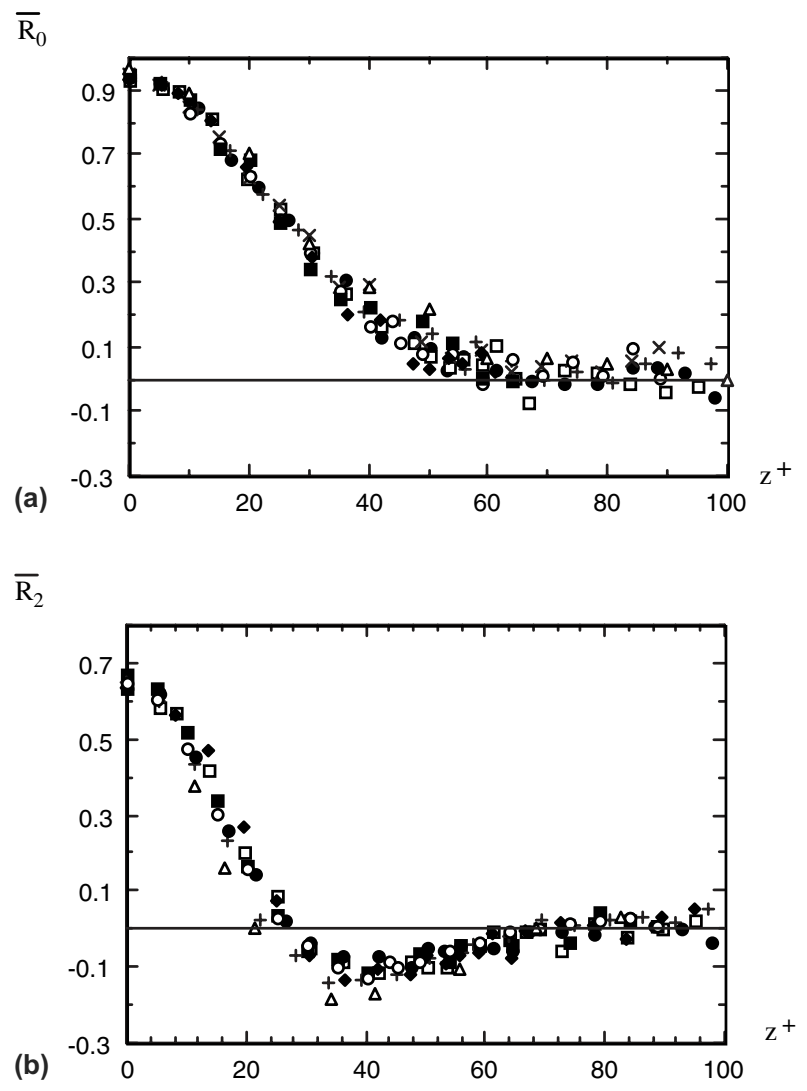

Fig. 9. Time mean spanwise correlation inferred from: (a) filter 0 and (b) filter 2. For legend see Fig. 3. 


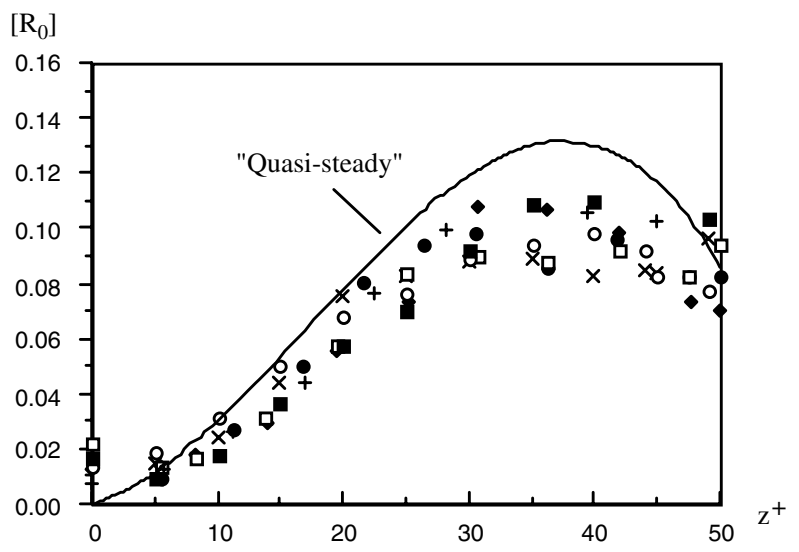

Fig. 10. The amplitude of the modulation of the correlation coefficient corresponding to the outer-layer structures. For legend see Fig. 3.

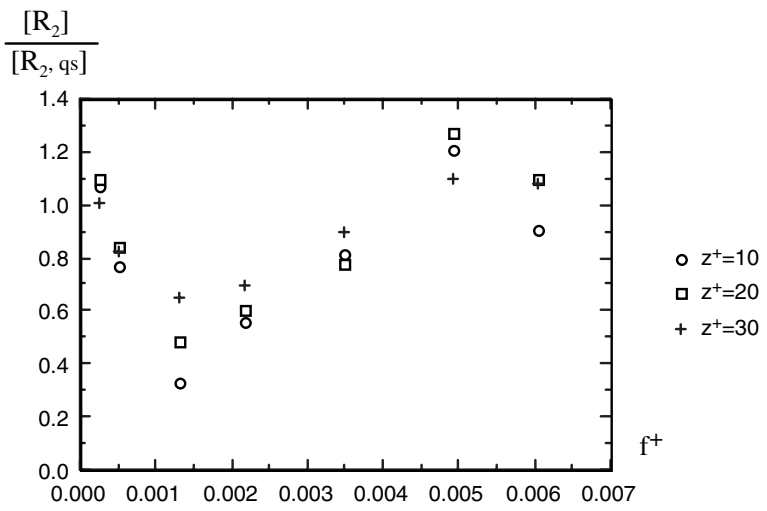

Fig. 11. Amplitude of the correlation coefficient obtained when filter 2 is used, compared with the quasi-steady amplitude versus the imposed frequency at three typical spanwise locations.

that $\left[R_{2}\right]$ decreases by a factor $2-3$ at the intermediate imposed frequency $f^{+}=0.0012$, in a way similar to the amplitude of the correlation coefficient of the unfiltered signals (Fig. 4b). This decay is followed by a subsequent increase in the high frequency regime showing that the inner structures are responsible for the true unsteady effects.

It would be more instructive at this step to consider the integral quantity defined by $\widehat{E}_{i}=\int_{0}^{z^{+}}\left[R_{i}\right] \mathrm{d} z^{+} /$ $\int_{0}^{z^{+}} \bar{R}_{i} \mathrm{~d} z^{+}$. One may show that $\widehat{E}_{i}$ represent the relative amplitudes of the integral scales to the first order (Vezin et al., 1994). Fig. 12 compares the distributions of $\widehat{E}_{i} / \widehat{E}_{i \mathrm{qs}}$ with the modulation of the $\langle R\rangle$ integral scale. It is seen that the response of the near wall eddies illustrated by $\widehat{E}_{1}$ and $\widehat{E}_{2}$ depends strongly on the imposed frequency. The reaction of the outer passive structures depicted through $\widehat{E}_{0}$ remains remarkably uniform.

\subsection{Modulation of streak spacing}

The modulation characteristics of the streak spacing are determined by computing the relative amplitude and
$\widehat{\mathrm{E}} / \widehat{\mathrm{E}}_{\mathrm{qs}}$ and $\widehat{\mathrm{E}}_{\mathrm{i}} / \widehat{\mathrm{E}}_{\mathrm{i}} \mathrm{qs}$

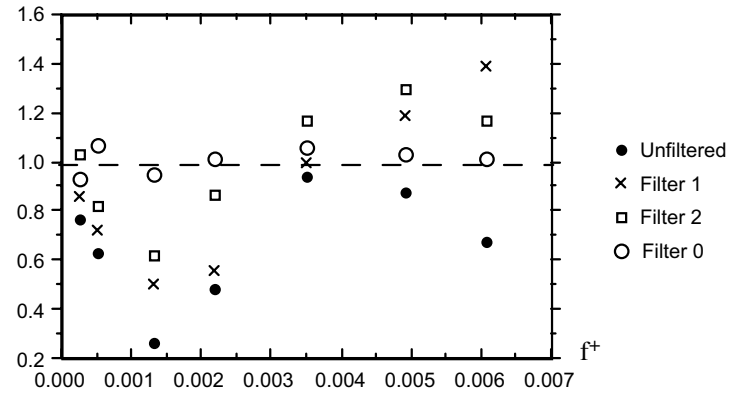

Fig. 12. Relative amplitudes of the pseudo integral scales (see the text) resulting from temporal filtering. Comparison between the reaction of outer and inner layer structures.

the phase of the zero crossing of the correlation coefficient. This is the procedure reported by Finnicum and Hanratty (1988) wherein the reasons of using such a scheme are discussed in detail.

The relative amplitude of the streak spacing scaled with the centerline amplitude $\hat{\lambda} / \hat{u}_{\mathrm{c}}$ is shown in Fig. 13a. The data is compared with the results inferred from the spanwise $\tau^{\prime}-\tau^{\prime}$ correlations reported by Finnicum and Hanratty (1988, FH hereafter). Consider first the quasisteady reaction of $\langle\lambda\rangle$. It is well established by now that the streak spacing scales with inner variables in steady flow (Smith and Metzler, 1983). One has therefore $\frac{\langle\lambda\rangle_{\mathrm{qs}}\left\langle u_{\tau}\right\rangle_{\mathrm{qs}}}{v} \approx \lambda^{+}$, which leads to $\hat{\lambda}_{\mathrm{qs}} \approx \hat{u}_{\mathrm{c}}$ and $\left\{\lambda_{\mathrm{qs}}\right\}-$ $\left\{u_{\mathrm{c}}\right\} \approx 180^{\circ}$. This is well established at $f^{+}<0.0005$ both for the measurements presented in this study and for those of FH (Fig. 13).
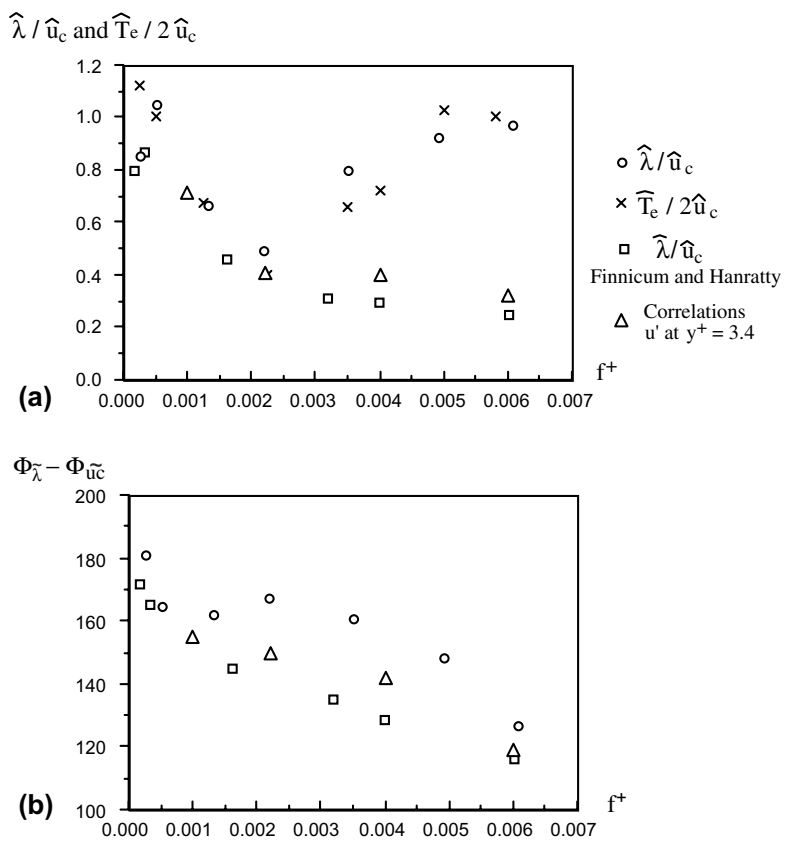

Fig. 13. Modulation characteristics of streak spacing vs. the imposed frequency: (a) amplitude; (b) phase shift. 
The streak spacing modulation relaxes first and $\hat{\lambda}$ decreases from the quasi-steady limit with a minimum near the critical frequency $f_{\mathrm{cr}}^{+}$. This is followed by an increase in the cyclic variations of $\langle\lambda\rangle$ in the high imposed frequency regime. The weakening of the streak spacing modulation at $f^{+}<f_{\text {cr }}^{+}$is in good agreement with FH. However, the subsequent increase of $\hat{\lambda}$ when $f^{+}>f_{\text {cr }}^{+}$does not agree. For all that, the two sets of data collapse quite satisfactorily as far as the phase shifts are concerned (Fig. 13b).

The longitudinal velocity fluctuations are instantaneously related to $\tau^{\prime}$ through $u^{\prime+}=\tau^{\prime+} y^{+}$only in the low viscous sublayer for $y^{+} \leqslant 2$ according to Popovich and Hummel (1967). Thus the disagreement between the present investigation related to the wall-buffer layer interactions and that of $\mathrm{FH}$ is, indeed, not surprising. To check this argument, we performed some correlation measurements $\left\langle\tau^{\prime} u^{\prime}\left(y^{+}=3.4\right)\right\rangle$, and deduced $\langle\lambda\rangle$. We could not go further closer to the wall, because of the well-known blocking effect. The triangles in Fig. 13 show that there is now a better agreement with FH. The effect of nonhomogeneity on the results raises the question of both the correct definition and determination of $\langle\lambda\rangle$ that physically represents the streak spacing modulation. This question is deferred to Section 4.1.

The amplitude and phase of the streak spacing obtained through flow visualizations by Brereton and Hwang (1994) are in profound disagreement with our measurements and those of FH. The ratio $\hat{\lambda} / \hat{u}_{\mathrm{c}}$ in their experiments varies between 1.2 and 1.8 at $f^{+}<0.0064$, and it is entirely out of range of the data presented here. More striking are the unexpected values of the phase shifts they report. In their investigation, $\{\lambda\}-\left\{u_{\mathrm{c}}\right\}$ decreases from $20^{\circ}$ in the low frequency regime (instead of $180^{\circ}$ that the quasi-steadiness would require) to -10 at $f^{+}=0.0064$. The shear-distortion length parameter they consequently introduced to scale the streak spacing, seems to infringe the quasi-steady rules. The very short record length $T_{\mathrm{r}}^{+}=6500$ in their experiments (which is 40 times smaller than ours) may be one of the causes of these controversies.

\subsection{Streak spacing and bursting}

The spanwise characteristic length in steady flow is related to the quiescent time period $T_{\mathrm{e}}$ during which the flow in the inner layer is relatively well ordered (Walker, 1990). $T_{\mathrm{e}}$ may be interpreted as the interval of time separating the energetic intermittent events, i.e. the ejections and sweeps. Consider the instantaneous behavior of the spanwise velocity $w^{\prime}$ at distances away from the wall where the viscous effects are negligible. The transient terms governing $w^{\prime}$ and related to the eddies that regenerate significant amount of $R e$ shear stress, must be of the same order of the convective terms, i.e. $\frac{\partial w^{\prime}}{\partial t} \sim w^{\prime} \frac{\partial w^{\prime}}{\partial z}$ according to Lyons et al., 1988.

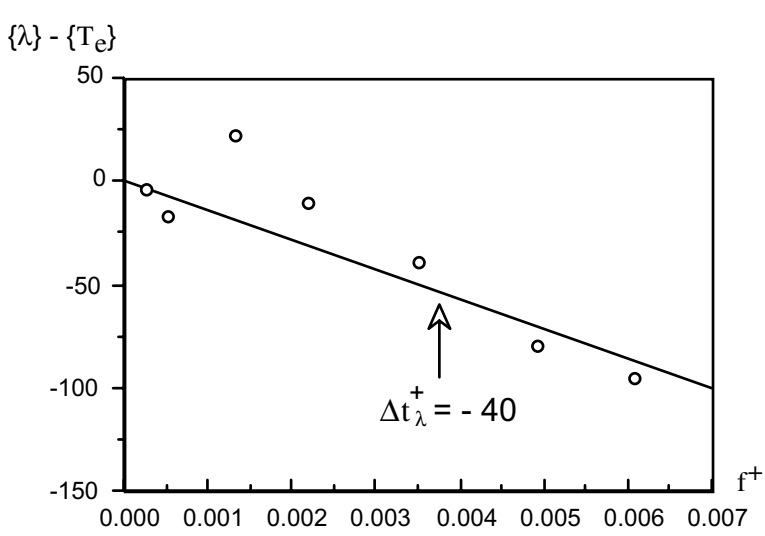

Fig. 14. Phase shift between the streak spacing and the ejection period.

This results in $\frac{\left\langle u_{\tau}\right\rangle_{\text {eff }}}{\left\langle T_{\mathrm{e}}\right\rangle} \sim \frac{\left\langle u_{\tau}\right\rangle_{\text {eff }}^{2}}{\langle\lambda\rangle}$, since the typical velocities near the wall scale with an effective shear velocity $\left\langle u_{\tau}\right\rangle_{\text {eff }}$ at a given time in the oscillation cycle. The phase averaged streak spacing, in return, scales with the effective inner variables by definition, and $\left\langle u_{\tau}\right\rangle_{\mathrm{eff}}=\frac{\langle\lambda\rangle}{v}=\bar{\lambda}^{+}$. Combining leads to $\left\langle T_{\mathrm{e}}\right\rangle \sim \frac{\langle\lambda\rangle^{2}}{v \lambda^{+}}$. This estimation shows that the relative amplitudes of the streak spacing and the ejection period should be linked through $\frac{1}{2} \widehat{T}_{\mathrm{e}} \approx \hat{\lambda}$ and that, one should have equality in the phases $\{\lambda\}$ and $\left\{T_{\mathrm{e}}\right\}$.

The Grenoble group investigated the reaction of the bursting mechanism in unsteady flows through well adapted one point detection schemes applied to $u^{\prime}(t)$ at $y^{+}=12$ (Tardu and Binder, 1997). The relative amplitude of the ejection period inferred from their study is shown in Fig. 13a. The ratio $\frac{T_{\mathrm{e}}}{2 \hat{u}_{\mathrm{c}}}$ is compared with $\frac{\hat{\lambda}}{\hat{u}_{\mathrm{c}}}$ in order to test the validity of the estimation given above. It is clearly seen that the two profiles collapse in an excellent manner in the whole imposed frequency range investigated here and that $\frac{1}{2} \widehat{T}_{\mathrm{e}} \approx \hat{\lambda}$. However, the phase shift shown $\{\lambda\}-\left\{T_{\hat{e}}\right\}$ in Fig. 14 is clearly not zero and decreases linearly with imposed frequency. The resulting time lag $\Delta t_{\lambda}^{+}=\frac{\{\lambda\}-\left\{T_{\mathrm{e}}\right\}}{2 \pi f^{+}}$is nearly constant with $\Delta t_{\lambda}^{+} \approx-40$. We give a tentative explanation of this time lag in Appendix B. Consequently, the estimation given before has to be rather replaced by $\left\langle T_{\mathrm{e}}(t)\right\rangle \sim \frac{\left\langle\lambda\left(t-\Delta t_{\lambda}\right)\right\rangle^{2}}{v \lambda^{+}}$. This result is quite attractive and indicates that the bursting and the spanwise streaky structure are linked by strong cause-to-effect relationships that the imposed nonequilibrium may hardly break down.

\section{Discussion}

\subsection{Physical meaning of the wall shear stress-streamwise velocity correlations}

Although the wall shear stress-streamwise velocity correlations have been used in steady flow to depict the 
near wall structure in a number of studies (Kreplin and Eckelmann, 1979; NW), no clear explanation has been given so far on the physical meaning of this quantity. An attempt is made here to give a more insight view of the results discussed in this paper and to explain the differences observed in $\langle\lambda\rangle$ obtained here and the streak spacing inferred from the wall shear stress correlations of $\mathrm{FH}$.

Wall shear stress-velocity correlations in forced unsteady flows will be derived here through a procedure similar to Hinze $(1975$, p. 328). The fluctuating wall shear stress is related to the fluctuating streamwise vorticity at the wall by the simple relationship $\tau^{\prime}(t)=$ $-\mu\left[\omega_{z}^{\prime}(t)\right]_{y=0}$. We will therefore consider the phaseaveraged correlation $\left\langle\left(\omega_{z}^{\prime}\right)_{\mathrm{A}}\left(u^{\prime}\right)_{\mathrm{B}}\right\rangle$, instead of $\left\langle\left(\tau^{\prime}\right)_{\mathrm{A}}\left(u^{\prime}\right)_{\mathrm{B}}\right\rangle$ (Fig. 15). The fluctuating spanwise vorticity at A diffuses according to

$\frac{\partial\left(\omega_{z}^{\prime}\right)_{\mathrm{A}}}{\partial t}=v \frac{\partial^{2}}{\partial x_{k} \partial x_{k}}\left(\omega^{\prime} z\right)_{\mathrm{A}}$

Therefore

$\frac{\partial\left(\tau^{\prime}\right)_{\mathrm{A}}}{\partial t}=v \frac{\partial^{2}}{\partial x_{k} \partial x_{k}}\left(\tau^{\prime}\right)_{\mathrm{A}}$

The streamwise velocity $u^{\prime}(t)$ at B is governed by

$$
\begin{aligned}
\left(\frac{\partial u^{\prime}}{\partial t}\right)_{\mathrm{B}}+\langle u\rangle_{\mathrm{B}}\left(\frac{\partial u^{\prime}}{\partial x}\right)_{\mathrm{B}}= & -\left(v^{\prime}\right)_{\mathrm{B}}\left(\frac{\partial\langle u\rangle}{\partial y}\right)_{\mathrm{B}} \\
& +\left[\frac{\partial}{\partial x_{k}}\left(\left\langle u^{\prime} u_{k}^{\prime}\right\rangle-u^{\prime} u_{k}^{\prime}\right)\right]_{\mathrm{B}} \\
& -\frac{1}{\rho}\left(\frac{\partial p^{\prime}}{\partial x}\right)_{\mathrm{B}}+v\left(\frac{\partial^{2} u^{\prime}}{\partial x_{k} \partial x_{k}}\right)_{\mathrm{B}}
\end{aligned}
$$

Mixed notations are used in (10) and (11) in order to alleviate Eqs. (Fig. 15). Because of the non-homogeneity of the turbulence in the wall normal direction $y=x_{2}$ and the imposed unsteadiness, any correlation $\langle Q\rangle=$ $\left\langle\left(q^{\prime}\left(t_{1}\right)_{\mathrm{A}}\right)\left(q^{\prime}\left(t_{2}\right)_{\mathrm{B}}\right)\right\rangle$ is a function of

$\langle Q\rangle=\langle Q\rangle\left[\zeta_{1}, \zeta_{2}, \zeta_{3},(y)_{\mathrm{AB}}, \frac{t_{1}+t_{2}}{2}, t_{2}-t_{1}\right]$,

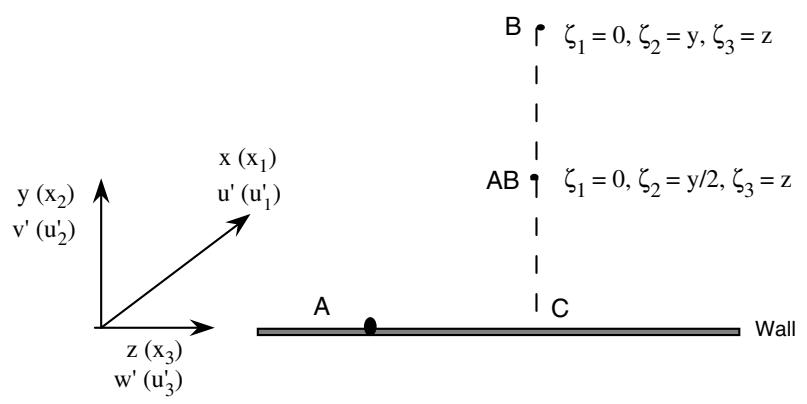

Fig. 15. Sketch of the configuration used to derive the wall shear stress-velocity correlations. where $\zeta_{k}=\left(x_{k}\right)_{\mathrm{B}}-\left(x_{k}\right)_{\mathrm{A}}$ and $(y)_{\mathrm{AB}}=\frac{1}{2}\left[(y)_{\mathrm{A}}+(y)_{\mathrm{B}}\right]$. Using these new variables, multiplying $(10)$ by $\left(u^{\prime}\right)_{\mathrm{B}},(11)$ by $\left(\tau^{\prime}\right)_{\mathrm{A}}$, adding these equations and finally phase averaging lead to

$$
\begin{aligned}
\frac{\partial}{\partial t}\left\langle Q_{3,1}\right\rangle+\langle u\rangle_{\mathrm{B}} \frac{\partial}{\partial \zeta_{1}}\left\langle Q_{3,1}\right\rangle= & -\left(\frac{\partial\langle u\rangle}{\partial y}\right)_{\mathrm{B}}\left\langle Q_{3,2}\right\rangle-\frac{\partial}{\partial \zeta_{k}}\left\langle S_{3,1 k}\right\rangle \\
& -\frac{1}{2}\left(\frac{\partial}{\partial y}\right)_{\mathrm{AB}}\left\langle S_{3,12}\right\rangle-\frac{1}{\rho} \frac{\partial}{\partial \zeta_{1}}\left\langle K_{3, p}\right\rangle \\
& +2 v \frac{\partial^{2}}{\partial \zeta_{k} \partial \zeta_{k}}\left\langle Q_{3,1}\right\rangle \\
& +\frac{1}{2} v\left(\frac{\partial^{2}}{\partial y \partial y}\right)_{\mathrm{AB}}\left\langle Q_{3,1}\right\rangle
\end{aligned}
$$

with the wall shear stress-velocity correlations: $\left\langle Q_{3,1}\right\rangle=\left\langle\left(\tau^{\prime}\right)_{\mathrm{A}}\left(u^{\prime}\right)_{\mathrm{B}}\right\rangle,\left\langle Q_{3,2}\right\rangle=\left\langle\left(\tau^{\prime}\right)_{\mathrm{A}}\left(v^{\prime}\right)_{\mathrm{B}}\right\rangle$; the wall shear stress-pressure correlation: $\left\langle K_{3, p}\right\rangle=\left\langle\left(\tau^{\prime}\right)_{\mathrm{A}}\left(p^{\prime}\right)_{\mathrm{B}}\right\rangle$, and the triple correlations: $\left\langle S_{3,1 k}\right\rangle=\left\langle\left(\tau^{\prime}\right)_{\mathrm{A}}\left(u^{\prime}\right)_{\mathrm{B}}\left(u^{\prime}\right)_{\mathrm{B}}\left(u_{k}^{\prime}\right)_{\mathrm{B}}\right\rangle$. The dynamic equation of $\widetilde{Q}_{3,1}$ is obtained by taking the timemean of the last equation, using the fact that $\bar{Q}_{3,1}$ is unaffected by the imposed unsteadiness and by subtracting the latter from (A.2.3). By the invariance under reflection, the second term at the left-hand side of (12) vanishes at $\zeta_{1}=0$. The result is

$$
\begin{aligned}
\frac{\partial}{\partial t} \widetilde{Q}_{3,1}= & -\left(\frac{\partial \bar{u}}{\partial y}\right)_{\mathrm{B}} \widetilde{Q}_{3,2}-\left(\frac{\partial \tilde{u}}{\partial y}\right)_{\mathrm{B}} \bar{Q}_{3,2}-\frac{\partial}{\partial \zeta_{k}} \widetilde{S}_{3,1 k} \\
& -\frac{1}{2}\left(\frac{\partial}{\partial y}\right)_{\mathrm{AB}} \widetilde{S}_{3,12}-\frac{1}{\rho} \frac{\partial}{\partial \zeta_{1}} \widetilde{K}_{3, p} \\
& +2 v \frac{\partial^{2}}{\partial \zeta_{k} \partial \zeta_{k}} \widetilde{Q}_{3,1}+\frac{1}{2} v\left(\frac{\partial^{2}}{\partial y \partial y}\right)_{\mathrm{AB}} \widetilde{Q}_{3,1}
\end{aligned}
$$

The derivatives with respect to $\zeta_{i}$ have to be computed at $\zeta_{1}=0, \zeta_{2}=y, \zeta_{3}=z$. The point $\mathrm{B}$ is at $y$ and $\mathrm{AB}$ is at $y / 2$ (Fig. 15). In a similar manner, the modulation of the $\tau^{\prime}-\tau^{\prime}$ correlations $\left\langle\Omega_{3,3}\right\rangle=\left\langle\left(\tau^{\prime}\right)_{\mathrm{A}}\left(\tau^{\prime}\right)_{\mathrm{C}}\right\rangle$ between $\mathrm{A}$ and $\mathrm{C}$ is simply

$\frac{\partial}{\partial t} \widetilde{\Omega}_{3,3}=2 v \frac{\partial^{2}}{\partial \zeta_{k} \partial \zeta_{k}} \widetilde{\Omega}_{3,3}+\frac{1}{2} v\left(\frac{\partial^{2}}{\partial y \partial y}\right)_{\mathrm{AC}} \widetilde{\Omega}_{3,3}$

The organized part of the wall shear stress-velocity correlations expressed in (13) can also be written in the form:

$$
\begin{aligned}
\frac{\partial}{\partial t} \widetilde{\tau_{\mathrm{A}}^{\prime} u_{\mathrm{B}}^{\prime}}= & -\left(\frac{\partial \bar{u}}{\partial y}\right)_{\mathrm{B}} \widetilde{\tau_{\mathrm{A}}^{\prime} v_{\mathrm{B}}^{\prime}}-\left(\frac{\partial \tilde{u}}{\partial y}\right)_{\mathrm{B}} \overline{\tau_{\mathrm{A}}^{\prime} v_{\mathrm{B}}^{\prime}} \\
& -\frac{1}{\rho} \tau_{A}^{\prime}\left(\frac{\widetilde{\partial p^{\prime}}}{\partial x}\right)_{\mathrm{B}}+\widetilde{T}+\widetilde{V}
\end{aligned}
$$

where $\widetilde{T}$ and $\widetilde{V}$ are respectively the triple nonlinear correlations, and the viscous terms. The first two quantities at the right hand side of (15) may be identified as production $\widetilde{P}$. These terms play an important role in the dynamics of the $\tau^{\prime}-u^{\prime}$ correlations. First of all, the 
wall normal fluctuating velocity $v^{\prime}$ is directly related to the streamwise vorticity $v^{\prime} \approx-\frac{1}{2} y^{2} \frac{\partial \omega_{x}}{\partial z}$ by near the wall and in the vicinity of the elongated streamwise structures (Jiménez and Moin, 1991). Therefore

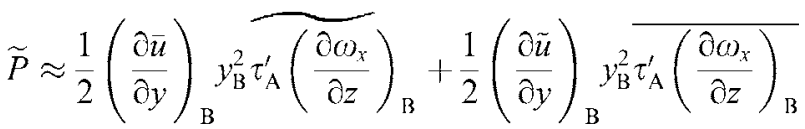

Clearly, $\tau^{\prime}-u^{\prime}$ is enhanced by spanwise variations of the quasi-streamwise structures, and this is nothing but the streak spacing. Secondly, $y_{\mathrm{B}}^{+}=10$ where the correlations are performed here, coincide with the bottom of the $\omega_{x}$ layers near the wall (Jiménéz, 1994). Consequently, the effect of the wall normal velocity they regenerate in the buffer layer is far being negligible.

The informations contained in the $\tau^{\prime}-u^{\prime}$ correlations performed in the buffer layer and connected to $\langle\lambda\rangle$ disappear progressively in the wall adjacent zone. Since $\widetilde{\tau_{\mathrm{A}}^{\prime} v_{\mathrm{B}}^{\prime}} \sim y_{\mathrm{B}}^{2}$ and $\widetilde{T} \sim y_{\mathrm{B}}^{2}$, the limiting form of the wall shear stress-velocity correlations as $y_{\mathrm{B} \rightarrow 0}$ is $\frac{\partial}{\partial t} \widetilde{\tau_{\mathrm{A}}^{\prime} u_{\mathrm{B}}^{\prime}}=$ $-\frac{1}{\rho} \widetilde{\tau_{\mathrm{A}}^{\prime}\left(\frac{\partial p^{\prime}}{\partial x}\right)}+\widetilde{V}$. Differentiating the last equation with respect to $y$ at $\mathrm{B}$, noting that in the wall adjacent layer $\partial p^{\prime} / \partial y \approx 0$ because $v^{\prime} \approx 0$ and finally that $\mu(\partial / \partial y)_{\mathrm{B}} u^{\prime} \approx$ $-\tau_{\mathrm{C}}^{\prime}$ as $y_{\mathrm{B}} \rightarrow 0$ (Fig. 15) one has $\frac{\partial}{\partial t} \tau_{\mathrm{A}}^{\prime} \tau_{\mathrm{C}}^{\prime}=\widetilde{V}^{\prime}$ where $\widetilde{V}^{\prime}$ is the corresponding viscous term. This equation is identical to that governing the spanwise correlations of the wall shear stress (Eq. 14). The $\tau^{\prime}-u^{\prime}$ correlations are similar to the $\tau^{\prime}-\tau^{\prime}$ correlations only very near the wall, where the production and pressure-vorticity interactions are both negligible. For these reasons alone, the streak spacing modulation detected directly at the wall, and through the structural correlations in the buffer layer are not necessarily identical. These arguments suggest that, the streak spacing modulation is well captured with the wall shear stress-velocity correlations in the low buffer layer.

\subsection{Conceptual model}

We introduced in Tardu and Binder (1997), a tentative conceptual model to explain the modulation characteristics of the ejection frequency. Arguments based on rapid distortion theory, the internal shear layers induced by convecting structures, and the vorticity generation mechanism suggested that the effective shear governing the near wall eddies is $\left\langle\alpha_{\text {eff }}\right\rangle / T_{\text {ID }}$ where the effective strain is governed by

$\frac{\partial\left\langle\alpha_{\mathrm{eff}}\right\rangle}{\partial t}=\frac{\partial\langle u\rangle}{\partial y}-\frac{\left\langle\alpha_{\mathrm{eff}}\right\rangle}{T_{\mathrm{ID}}}$

because the turbulence has only limited time to maintain an equilibrium structure. The streak spacing modulation is given by $\frac{\left\langle\alpha_{\text {eff }}\right\rangle}{T_{\mathrm{ID}}}\langle\lambda\rangle^{2}=\frac{\partial \bar{u}}{\partial y} \bar{\lambda}^{2}$

which leads to a correct form in the quasi-steady regime.

The integral distortion time $T_{\mathrm{ID}}$ takes into account the history of distortion over the lifetime of the eddies, rather than the local effective distortion used in unsteady closures (Mankbadi and Liu, 1992; Tardu and Da Costa, 2001). An eddy originating from the wall sees different kinds and rates of strain and it is its history that matters for the development of near wall structures, including the streaks. The effective duration of the straining is limited by the average lifetime of the structure, which is nothing but the ejection period $T_{\mathrm{e}}^{+}$. Consider first the high frequency regime wherein the oscillating shear is confined into $y^{+} \leqslant l_{\mathrm{s}}^{+}$. The eddy interacts with the unsteady shear during a "residence time" $T_{\mathrm{r}}$ in the Stokes layer, grows up, achieves its typical final form at a distance $d^{+}=20$ over the wall (Jiménéz, 1994) and disintegrates at $t^{+}=T_{\mathrm{e}}^{+}$. Assuming that the growth rate is uniform, it is conjectured that $T_{\mathrm{r}}^{+} \approx \frac{l_{\mathrm{s}}^{+}}{d^{+}} T_{\mathrm{e}}^{+}$which is also approximately equal to the time period during which the eddy has been directly distorted by the oscillating shear, i.e. $T_{\mathrm{ID}}^{+}=T_{\mathrm{r}}^{+}$. This is valid when the residence time of the eddy is small compared with its lifetime $T_{\mathrm{r}}^{+}<T_{\mathrm{e}}^{+}$, or equivalently when $l_{\mathrm{s}}^{+}<d^{+}$. When, in return, $l_{\mathrm{s}}^{+}>d^{+}$the eddy is continuously distorted by the oscillating shear during its entire lifetime and consequently $T_{\mathrm{ID}}^{+}=T_{\mathrm{e}}^{+}$. Combining these arguments with (17) and (18), one has for small imposed amplitudes:

$$
\begin{aligned}
& l_{\mathrm{s}}^{+} \geqslant 20, \quad 2 \frac{\hat{\lambda}}{\hat{\tau}}=\frac{1}{\sqrt{1+\left(2 \pi f^{+} T_{\mathrm{e}}^{+}\right)^{2}}}, \\
& \{\lambda\}-\{\tau\}=\pi-\operatorname{arctg}\left(2 \pi f^{+} T_{\mathrm{e}}^{+}\right) \quad \text { with } T_{\mathrm{e}}^{+}=100 \\
& l_{\mathrm{s}}^{+}<20, \quad 2 \frac{\hat{\lambda}}{\hat{\tau}}=\frac{1}{\sqrt{1+\left(\frac{2 \sqrt{\pi f^{+}}}{d^{+}} T_{\mathrm{e}}^{+}\right)^{2}}}, \\
& \{\lambda\}-\{\tau\}=\pi-\operatorname{arctg}\left(\frac{2 \sqrt{\pi f^{+}}}{d^{+}} T_{\mathrm{e}}^{+}\right)
\end{aligned}
$$

The time mean ejection period in (19) is typically $T_{\mathrm{e}}^{+}=100$ in the low buffer layer (Tardu, 2002). It is seen in Fig. 16 that there is a reasonable agreement of the model with the experiments, especially for the amplitudes. The phase shifts however agree only qualitatively.

These arguments are presumably valid, if the identification procedure is capable of detecting the entire regeneration process of $R e$-shear stress producing eddies in the buffer layer. The detection in the low viscous sublayer or directly at the wall is disconnected with the rich information contained in the buffer layer as discussed in the previous session. There is, in this case, a direct effect of the oscillating shear and of the smaller structures that develop in the viscous sublayer as 


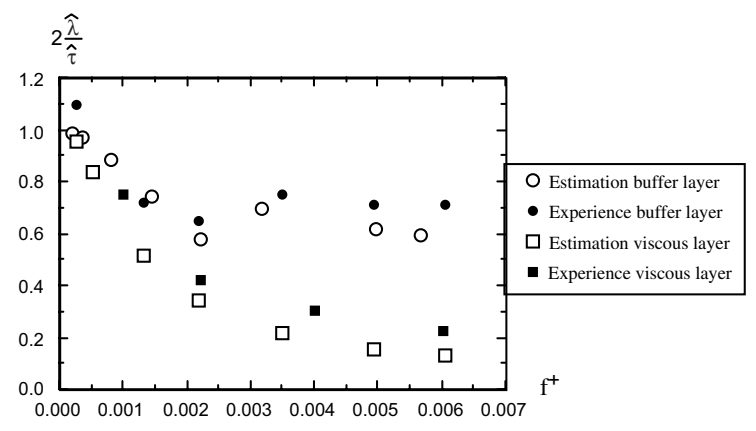

$\{\lambda\}-\{\tau\}$

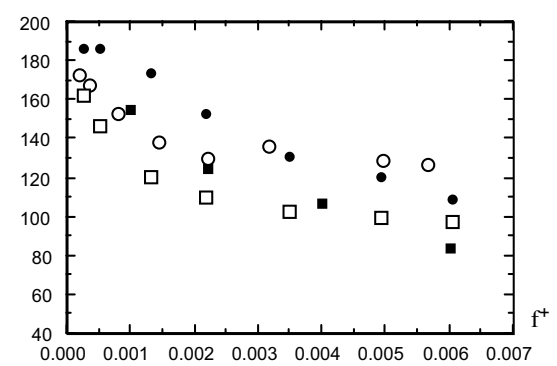

Fig. 16. Confrontation of the conceptual model with the experiments.

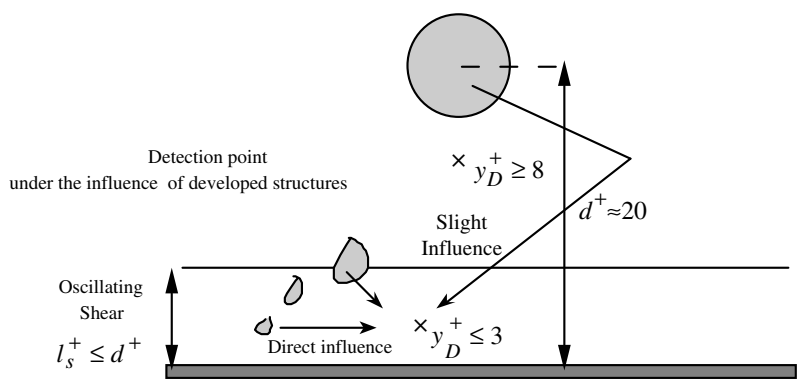

Fig. 17. Conceptual model for detection in the buffer and low viscous sublayers.

schematically shown in Fig. 17. The structures that regenerate the streaks are always in the oscillating shear zone and those that quit this zone have only negligible influence because of finite response time in the high imposed frequency regime. The modulation characteristics of the streak spacing, should, under these circumstances, be rather governed by the local lifetime of the structures, i.e. the local ejection period which is $T_{\mathrm{e}}^{+} \approx 200$ in the low viscous sublayer (Tardu, 2002). Thus, considering that the detection point is always in the oscillating shear region one should have

$2 \frac{\hat{\lambda}}{\hat{\tau}}=\frac{1}{\sqrt{1+\left(2 \pi f^{+} T_{\mathrm{e}}^{+}\right)^{2}}}$,

$\{\lambda\}-\{\tau\}=\pi-\operatorname{arctg}\left(2 \pi f^{+} T_{\mathrm{e}}^{+}\right)$with $T_{\mathrm{e}}^{+}=200$

We confront in Fig. 16 the experiments to (20) that shows a satisfactory agreement, considering the crudeness of the model.

\section{Conclusion}

The results reported in this study are reminiscent of the important role played by the inner structures in the unsteady response of the near wall turbulence. The spanwise correlation coefficient corresponding to the outer structures, responds uniformly to the imposed unsteadiness, without any manifestation of relaxation. At the same time, the contribution of the outer eddies to $\left\langle u^{\prime} u^{\prime}\right\rangle$ and $\left\langle\tau^{\prime} \tau^{\prime}\right\rangle$ lags the centerline velocity with a constant response-time, while the frequency-response characteristics of the inner structures are altered at high $f^{+}$. The changes in the time lags of the modulation of the fluctuating quantities in the high imposed frequency range occur when the imposed period is of the same order of the time scales of the inner structures. This happens roughly, when the oscillating shear becomes confined into the low buffer layer. These results may have important consequences in the modeling of the near wall unsteady flows, and suggest the insertion of constant and frequency dependent response times for the outer and the inner structures respectively.

It is found that the relative amplitudes of the ejection period and of the streak spacing have the same qualitative and quantitative distributions versus the imposed frequency when they are properly scaled. The modulation of the streak spacing lags $\left\langle T_{\mathrm{e}}\right\rangle$ by about 40 wall units. There is a reasonable agreement between the experiments and a conceptual model that takes into account the time history of distortion over the lifetime of the structures. When confirmed by further studies, these results which are relatively new in the topic of unsteady flows, would indicate a close link between the streaky spanwise structure and the energetic active wall events. The direct and the large eddy simulations would largely contribute to a better understanding of these complex flows, in particular through the analysis of the vorticity dynamics.

\section{Acknowledgements}

We thank warmly D. Ronneberger for helpful discussions on the spectral decomposition of the unsteady processes. Financial support of the Délégation Générale de l'Armement via the Direction des Recherches, Etudes et Techniques (Contract no. 91.086) is gratefully acknowledged.

\section{Appendix A. Response of the wall-hot film gages}

The wall shear stress measurements are delicate under certain circumstances and the frequency response of the wall hot-film gages may be poor because of the effect of the conduction into the substrate. This effect is negligible in the present working conditions (glass-substrate/ water-fluid combination). The attenuation of the fre- 
Table 1

Time mean statistics of the turbulent fluctuations of the wall shear stress in steady and unsteady flow: comparison with existing data

\begin{tabular}{clll}
\hline & $\begin{array}{l}\text { Steady flow, } \\
\text { this study }\end{array}$ & $\begin{array}{l}\text { Steady flow, } \\
\text { Kim et al. } \\
(1987)\end{array}$ & $\begin{array}{l}\text { Unsteady flow, } \\
\text { this study }\end{array}$ \\
\hline$\sqrt{\frac{\overline{\tau^{\prime} \tau^{\prime}}}{\bar{\tau}}}$ & 0.38 & 0.36 & $0.36-0.41$ \\
$S_{\tau^{\prime}}=\frac{\overline{\tau^{3}}}{\overline{\tau^{\prime 2 / 2}}}$ & 1.15 & 1.00 & $0.95-1.25$ \\
$F_{\tau^{\prime}}=\frac{\overline{\tau^{\prime}}}{\tau^{2^{2}}}$ & 4.20 & 4.00 & $3.80-4.20$ \\
\hline
\end{tabular}

quency response is less than $4 \%$ as given by the computations of the full heat transfer problem carried out by Tardu et al. (1991).

The quality of the wall shear stress measurements was checked by comparing the statistics of the fluctuating wall shear stress $\tau^{\prime}(t)$ in steady canonical channel flow with existing data. The results obtained at $R e_{\mathrm{h}}=8800$ are summarized in Table 1. It is seen that $\sqrt{\tau^{\prime} \tau^{\prime}}$ is 0.38 times the wall shear stress $\bar{\tau}$ in agreement with 0.36 found from the direct numerical simulation data of Kim et al. (1987). The high order statistics, i.e. the skewness $S_{\tau^{\prime}}=\frac{\overline{\tau^{\prime}}}{\overline{\tau^{\prime 2}}}$ and the flatness $F_{\tau^{\prime}}=\frac{\overline{\tau^{4}}}{{\overline{\tau^{\prime 2}}}^{2}}$ of $\tau^{\prime}(t)$ were also measured. It was found that, in steady flow, $S_{\tau^{\prime}}=1.15$ and $F_{\tau^{\prime}}=4.20$. These values agree also well with Kim et al. (1987). The time mean statistics in unsteady flow are shown in the last column of Table 1 . No trend was observed as the frequency varied, thus confirming the insensitivity of the mean flow to imposed unsteadiness.

The frequency response of the HWG was further checked by comparing the modulation characteristics of $\left\langle\tau^{\prime} \tau^{\prime}\right\rangle$ with those of the fluctuating longitudinal velocity $\left\langle u^{\prime} u^{\prime}\right\rangle$ measured at $y^{+}=2.5$, by LDA (Tardu et al., 1994). Good correspondence is found between $\frac{\sqrt{\left\langle\tau^{\prime} \tau^{\prime}\right\rangle}}{\langle\tau\rangle}$ and $\frac{\sqrt{\left\langle u^{\prime} u^{\prime}\right\rangle}}{\langle u\rangle}$, and the resulting amplitudes and phases differed by less than $5 \%$. These ratios are only asymptotically equal as $y^{+} \rightarrow 0$, but it was not possible to make accurate measurements with LDA nearer than $y^{+}=2.5$ to the wall.

\section{Appendix B. Wigner-Ville spectrum and cyclostationary processes}

We used well-established characteristics of cylostationary processes $(\mathrm{CP})$ in this paper to determine the response of outer and inner eddies. The use of WignerVille spectrum is of course allowed, since it gives information in time-frequency domain, yet the Wigner-Ville spectrum has to be connected with the properties of $\mathrm{CP}$.

The Wigner-Ville spectrum is defined as

$$
W_{x}(t, \eta)=\int_{-\infty}^{+\infty} \Phi_{x}\left(\eta-\frac{\zeta}{2}, \eta+\frac{\zeta}{2}\right) \mathrm{e}^{-j 2 \pi \zeta t} \mathrm{~d} \zeta
$$

The support of $\Phi_{x}$ is

$f_{1}=f_{2}-\frac{i}{T}, \quad i \in Z$

because $x$ is cyclostationary. Therefore

$W_{x}(t, \eta)=\int_{-\infty}^{+\infty} \Phi_{x}\left(\eta-\frac{\zeta}{2}, \eta+\frac{\zeta}{2}\right) \mathrm{e}^{-j 2 \pi \zeta t} \delta\left(\zeta-\frac{i}{T}\right) \mathrm{d} \zeta$

where $\delta$ is the Dirac function. It is seen that the WienerVille spectrum can be expressed in discrete form in such cases with the $i$ th component being

$W_{x i}(t, v)=\Phi_{x}\left(v-\frac{i}{2 T}, v+\frac{i}{2 T}\right) \mathrm{e}^{-j \frac{2 \pi i t}{T}}$

The component $i=0$, for instance is the stationary part of the process, i.e.

$W_{x 0}(t, v)=\Phi_{x}(v, v)$

The Wiener-Ville spectrum of a CP process is consequently given by

$W_{x}(t, v)=\sum_{i \in Z} \Phi_{x}\left(v-\frac{i}{2 T}, v+\frac{i}{2 T}\right) \mathrm{e}^{-j \frac{2 \pi i t}{T}}$

Fig. 18 shows the domain recovered by the Wigner-Ville spectrum for an octave pass-band filter $[f, 2 f]$ when $\eta=\frac{3}{2} f$. The integration domain of Eq. (A.2.1) is limited to the line $f_{2}=2 \eta-f_{1}=3 f-f_{1}$. It is seen that only a limited countable number of points contribute to $W_{x}(t, \eta)$. The output of the pass band filter as used here is related to $W_{x}(t, \eta)$ through the integral:

$\langle x x\rangle=\int_{\eta=f}^{\eta=2 f} W_{x}(t, \eta) \mathrm{d} \eta$

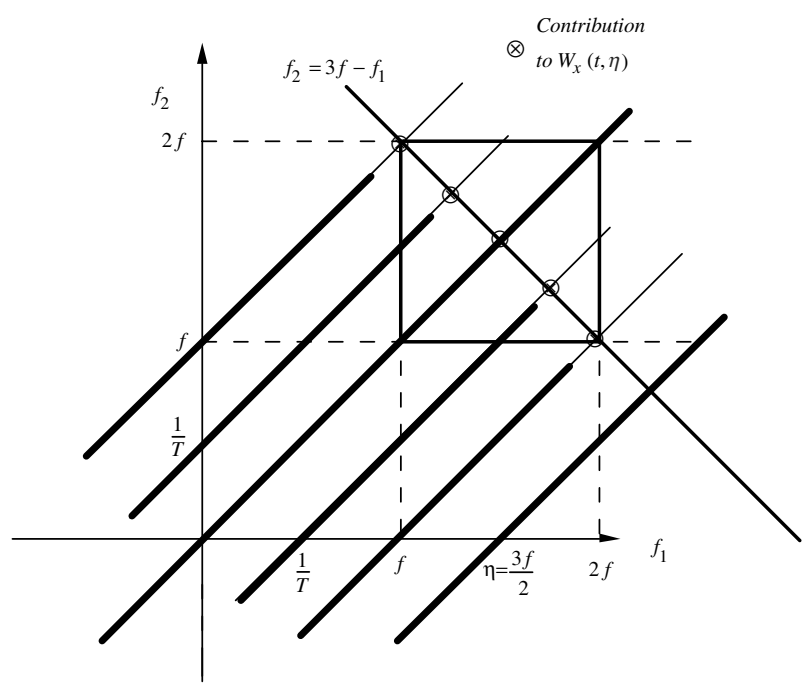

Fig. 18. Spectral domain recovered for the Wigner-Ville spectrum $W_{x}(t, \eta)$ in a octave pass band filter centred at $\eta=\frac{3}{2} f$ for a cyclostationary process $x(t)$. The spectral support shown by the bold lines can be distributed in such a way that the modulation $\widetilde{W}_{x}(t, \eta)$ is zero, but not $\langle x x\rangle$ in the band covered by the filter its integral. 


\section{Appendix C. Plausible cause-to-effect relationships be- tween the ejection frequency and the streak spacing}

We will anticipate a plausible explanation of the time lag $\Delta t_{\lambda}^{+}$discussed in Section 3.6, by adapting the simplified cycle for the near wall events proposed by Jiménéz (1994) and shown schematically in Fig. 19.

(i) The model starts with a perturbation that produces a streamwise dependence in the low speed streaks with an initial spacing $\left\langle\lambda\left(t_{0}\right)\right\rangle$ at the time $t_{0}$ in the oscillation cycle. The streaks are modeled as co-flowing jets and the streamwise scale of the perturbation is selected as a multiple of their width. That results in a vertical slab of normal vorticity with a thickness proportional to $\left\langle\lambda\left(t_{0}\right)\right\rangle$ and which is tilted by the phase averaged effective shear. At a later stage the tilting becomes stretching, resulting in a layer of streamwise vorticity. The long time limit of this process is a Burger's vortex sheet that is axially strained and diffuses. The short time limit is the advection by the mean shear. Both trends coincide in a set-up time related to the initial streak spacing by
$\Delta t_{\mathrm{s}}^{*} \approx \frac{1}{2}\left\langle\lambda^{*}\left(t_{0}\right)\right\rangle^{2 / 3}$

This relationship is the "unsteady version" of $\overline{\Delta t_{s}^{+}} \approx$ $\frac{1}{2} \bar{\lambda}^{+2 / 3}$ given by Jiménez in steady flow (1994, p. 947). The asterisk stands for scaling with $v$ and $\left\langle u_{\tau}\right\rangle_{\text {eff }}$. The unsteadiness is formally taken into account by substituting the time mean values by the scaled phase averaged ones. The set-up time is roughly $\Delta t_{\mathrm{s}}^{+} \approx 9$ for the most probable streak spacing $\bar{\lambda}^{+} \approx 80$. Prior to its set-up, the layer of streamwise vorticity rolls up into a quasistreamwise vortex (QSV) in approximately half an eddy turnover time (Orlandi and Jiménez, 1991). The typical value of $\Delta t_{\mathrm{r}}$ in wall units is $\Delta t_{\mathrm{r}}^{+}=\frac{\left(2 \pi r^{+}\right)^{2}}{2 \Gamma^{+}} \approx 10$ where $r_{0}^{+} \approx 10$ and $\Gamma^{+} \approx 200$ are respectively the radius and circulation of the QSV's in the near wall region. The time interval separating the perturbed initial streak and the newly generated QSV is therefore $\Delta t_{\mathrm{F}}^{+}=\Delta t_{\mathrm{s}}^{+}+\Delta t_{\mathrm{r}}^{+} \approx 19$ and this initial stage is quite rapid.

(ii) The circulation of the new quasi-streamwise vortex comes from the "one contained in the initial
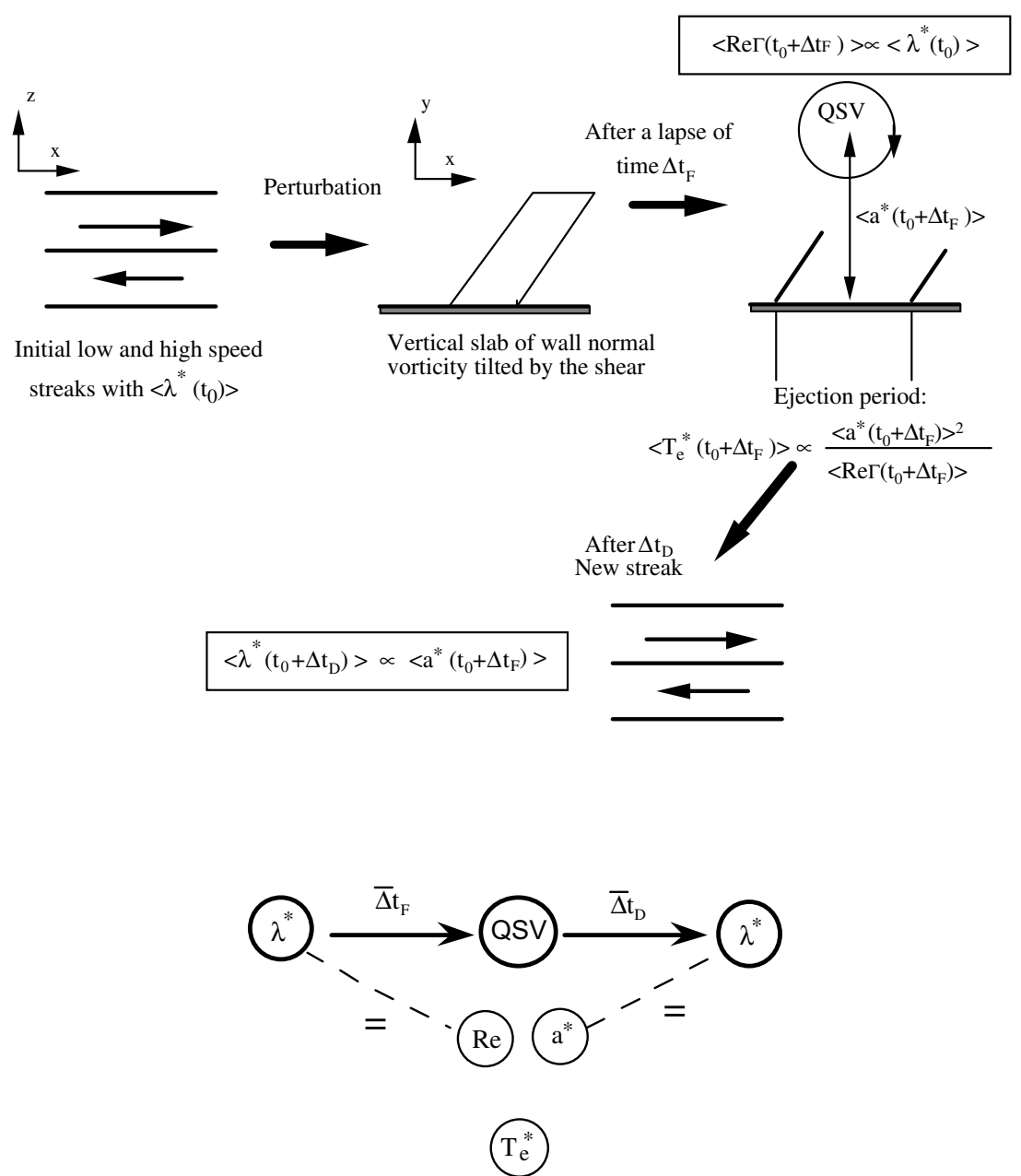

Fig. 19. Chain of events in the genesis of near wall structures according to Jiménéz (1994). 
perturbation which is proportional to the width of the (initial) streak". The associated Reynolds number of the QSV is consequently related to the "past history" by1

$\left\langle\operatorname{Re}_{\Gamma} \cdot\left(t_{0}+\Delta t_{\mathrm{F}}\right)\right\rangle=\langle\Gamma\rangle / 2 \pi v \propto\left\langle\lambda^{*}\left(t_{0}\right)\right\rangle$

Jiménézp. 947 (1994) argues further that, the distance of the new QSV to the wall determines the width of its own streak, i.e.:

$\left\langle\lambda^{*}\left(t_{0}+\Delta t_{\mathrm{D}}\right)\right\rangle \propto\left\langle a^{*}\left(t_{0}+\Delta t_{\mathrm{F}}\right)\right\rangle$

where $\Delta t_{\mathrm{D}}$ stands for the transient development time of the new streaky structure. According to Walker (1990), $\Delta t_{\mathrm{D}}$ scales with the streak spacing and the average magnitude $w_{\mathrm{c}}^{+} \approx 2$ of the characteristic spanwise velocity during the quiescent period. A rough estimate of the development time is therefore $\Delta t_{\mathrm{D}}^{+} \approx \frac{\lambda^{+}}{w_{\mathrm{c}}^{+}} \approx 40$.

(iii) The eruptive response of the near wall layer under the influence of the QSV, i.e. the occurrence of the ejections, depends upon its distance to the wall $a^{+}$and its intensity $\operatorname{Re}_{\Gamma}$. The time scale of the eruptive response in steady flow is

$\bar{T}_{\mathrm{e}}^{+} \propto \frac{\bar{a}^{+2}}{\overline{\operatorname{Re}}_{\Gamma}}$

according to Smith et al. (1991). In other words, the stronger the structures, or the closer they are to the wall, the smaller is the inter-arrival time between ejections. Note that, the estimations $\bar{a}^{+} \propto \bar{\lambda}^{+}$and $\overline{R e}_{\Gamma} \propto \bar{\lambda}^{+}$given in steady flow by Jiménez and combined with (4) leads to $\bar{T}_{\mathrm{e}}^{+} \propto=80$ which is in agreement with the experiments (Tiedermann, 1988). Accordingly, the ejection period at $t_{0}+\Delta t_{\mathrm{F}}$ in our case is

$$
\left\langle T_{\mathrm{e}}^{*}\left(t_{0}+\Delta t_{\mathrm{F}}\right)\right\rangle \propto \frac{\left\langle a^{*}\left(t_{0}+\Delta t_{\mathrm{F}}\right)\right\rangle^{2}}{\left\langle\operatorname{Re}_{\Gamma}\left(t_{0}+\Delta t_{\mathrm{F}}\right)\right\rangle} \propto \frac{\left\langle\lambda^{*}\left(t_{0}+\Delta t_{\mathrm{D}}\right)\right\rangle^{2}}{\left\langle\lambda^{*}\left(t_{0}\right)\right\rangle}
$$

obtained by combining (A.3.2) and (A.3.3) and using the unsteady version of (A.3.4). A Taylor series to the first order leads to

$$
\begin{aligned}
\left\langle T_{\mathrm{e}}^{*}\left(t_{0}+\Delta t_{\mathrm{F}}\right)\right\rangle & \propto\left\langle\lambda^{*}\left(t_{0}\right)\right\rangle\left[1+2 \frac{\partial\left\langle\lambda^{*}\right\rangle}{\partial t} \frac{\Delta t_{\mathrm{D}}}{\left\langle\lambda^{*}\left(t_{0}\right)\right\rangle}\right] \\
& \approx\left\langle\lambda^{*}\left(t_{0}+2 \Delta t_{\mathrm{D}}\right)\right.
\end{aligned}
$$

or equivalently

$\left\langle T_{\mathrm{e}}^{*}(t)\right\rangle \propto\left\langle\left(t-\Delta t_{\lambda}^{\sim}\right)\right\rangle$

where $\Delta t_{\lambda}^{\sim} \approx-\left[2 \Delta t_{\mathrm{D}}-\Delta t_{\mathrm{F}}\right]$. Eq. (A.3.7) is reminiscent of a time shift between the ejection period and the streak spacing. By making use of the estimations given before, one obtains a time lag $\Delta t_{\lambda}^{+} \approx-\left[2 \Delta t_{\mathrm{D}}^{+}-\Delta t_{\mathrm{F}}^{+}\right] \approx-61$ in wall units. Considering the crudeness of the model, this is in fair agreement with $\Delta t_{\lambda}^{+} \approx-40$ observed in Fig. 14. It must be emphasized that this result is the consequence of a well-ordered cycle of events, and therefore not coincidental.

\section{References}

Beykirch, M., Hesse, M.K., Ronneberger, D., 1996. The unsteady spectral properties of turbulent channel flow at time variant boundary conditions. In: Gavrilakis, et al. (Eds.), Advances in Turbulence VI, pp. 513-514.

Bradshaw, P., 1967. Inactive motion and pressure fluctuations in turbulent boundary layers. J. Fluid Mech. 30, 241.

Brereton, G.-J., Reynolds, W.-C., Jarayaman, R., 1990. Response of a turbulent boundary layer to sinusoidal free-stream unsteadiness. J. Fluid Mech. 221, 131.

Brereton, G.-J., Hwang, J.-L., 1994. The spacing of streaks in unsteady turbulent wall-bounded flow. Phys. Fluids 6 (7), 2446.

Brereton G.J., Mankbadi R.R., 1993. A rapid distortion theory turbulence model for developed unsteady wall-bounded flows. In: International Conference on Near-Wall Turbulent Flows, Tempe, Arizona.

Feng, M.-Q., Tardu, S.-F., Binder, G., 1993. Inner region of an unsteady channel flow. In: Proc. Near Wall Turbulence. Elsevier Pub. pp. 457-466.

Finnicum, D.-S., Hanratty, T.-J., 1988. Effect of imposed sinusoidal oscillations on turbulent flow in a pipe. PCH 10, 585.

Flandrin, P., 1998. Temps-fréquence. Editions Hermes, Paris

Gardner, W.-A., Franks, L.-E., 1975. Characterization of cyclostationary random signal processes. IEEE Trans. Information Theory IT-21, 4-14.

Hurd, H.-L., 1989. Representation of strongly harmonizable periodically correlated processes and their covariances. J. Multivariate Anal. 29, 53-67.

Hinze, J.E., 1975. Turbulence. McGraw-Hill Series, New York.

Jiménez, J., Moin, K., 1991. The minimal flow unit in near wall turbulence. J. Fluid Mech. 225, 213.

Jiménéz, J., 1994. On the structure and control of near wall turbulence. Phys. Fluids 6, 944.

Kim, J., Moin, P., Moser, R., 1987. Turbulence statistics in fully developed channel flow at low Reynolds number. J. Fluid Mech. 177, 133.

Kreplin, H.-P., Eckelmann, H., 1979. Propagation of perturbations in the viscous sublayer and adjacent wall region. J. Fluid Mech. 95, 305.

Lyons, S.-L., Nikolaides, C., Hanratty, T.-J., 1988. The size of turbulent eddies close to a wall. AIChE J. 34, 938.

Mankbadi, R.-R., Liu, J.-T.-L., 1992. Near wall response in turbulent shear flows subjected to imposed unsteadiness. J. Fluid Mech. 238, 55.

Mao, Z.-X., Hanratty, T.-J., 1986. Studies of wall shear stress in a turbulent pulsating pipe flow. J. Fluid Mech. 177, 454.

Maxey, M.R., 1982. Distortion of turbulence in flows with parallel streamlines. J. Fluid Mech. 124, 261.

Naguib, A.-M., Wark, C.-E., 1992. An investigation of wall-layer dynamics using a combined temporal filtering and correlation technique. J. Fluid Mech. 243, 541.

Orlandi, P., Jiménez, J., 1991. A model for bursting of near wall vortical structures in boundary layers. Proceedings of the Eight Symposium on Turbulent Shear Flows; Technical University of Munich, Germany, pp. 2811-2816.

Popovich, A.-T., Hummel, R.-L., 1967. AIChE J 13, 854

Ronneberger, D., Ahrens, C.D., 1977. Wall shear stress caused by signal amplitude perturbations of turbulent boundary-layer flow: an experimental investigation. J. Fluid Mech. 83, 433.

Smith, C.-R., Metzler, S.-P., 1983. The characteristics of low-speed streaks in the near-wall region of a turbulent boundary layer. J. Fluid Mech. 129, 27. 
Smith, C.R., Walker, J.D.A., Haidari, A.H., Sobrun, U., 1991. On the dynamics of near wall turbulence. Philos. Trans. R. Soc. London Ser. A 336, 131-175.

Tardu, S.-F., Pham, C.-T., Binder, G., 1991. Effects of longitudinal diffusion in the fluid and heat conduction to the substrate on the response of wall-hot film gages. In: Johannson, A.-V., Alfredsson, P.-H. (Eds.), Advances in Turbulence III. Springer-Verlag, pp. 506-513.

Tardu, S.-F., Binder, G., 1993. Response of turbulence to imposed oscillations of high frequencies. Phys. Fluids A 5 (8), 2028.

Tardu, S.-F., Binder, G., Blackwelder, R.-F., 1994. Turbulent channel flow with large amplitude velocity oscillations. J. Fluid Mech. 267, 109.

Tardu, S.-F., Binder, G., 1997. Reaction of bursting to an homogeneous oscillating pressure gradient. Eur. J. Mech. B, 1-34.

Tardu, S.-F., Da Costa, P., 2001. Modeling of unsteady wall flows with and without adverse pressure gradient by a $k-\omega /$ rapid distorsion closure. Second International Turbulent Shear Flow Phenomena Symposium, Stockholm, June 27-29.

Tardu, S.-F., 2002. Characteristics of single and multiple bursting events. Part 2: level crossing events. Exp. Fluids 33, 640.

Tardu, S.F., 2003. Caractérisation des écoulements turbulents périodiques. Comptes Rendus de l'Académie des Sciences 331, 767774.

Tiedermann, W.-G., 1988. Eulerian detection of turbulent bursts. In: Proceedings of Zaric Intern. Sypm. on Near Wall Turbulence.

Tu, S.-W., Ramaprian, B.-R., 1983. Fully developed periodic turbulent pipe flow. J. Fluid Mech. 137, 31.

Vezin, P., Tardu, S.-F., Binder, G., 1994. Corrélations spatio-temporelles et échelles de longueur dans les écoulements pulsés en canal. Rapport de Synthèse Finale no. 91.086.

Walker, J.D.-A., 1990. Models based on dynamical features of the wall layer. Appl. Mech. Rev. 43, S232. 